\title{
Folheações infinitesimalmente polares
}

\author{
Rafael Briquet
}

Dissertação apresentada

aO

INSTITUTO DE MATEMÁTICA E ESTATÍSTICA

da

UNIVERSIDADE DE SÃO PAULO

para

obtenção do título

de

Mestre em Ciências.

Programa: Matemática.

Orientador: Prof Dr Marcos Martins Alexandrino da Silva.

Durante o desenvolvimento deste trabalho

o autor recebeu auxílio financeiro do CNPq.

São Paulo, abril de 2011. 



\title{
Folheações infinitesimalmente polares
}

\author{
Esta dissertação contém as correções e alterações \\ sugeridas pela Comissão Julgadora durante a defesa \\ realizada por Rafael Briquet em 29/04/2011. \\ O original encontra-se disponível no \\ Instituto de Matemática e Estatística \\ da Universidade de São Paulo.
}

Comissão Julgadora:

- Prof Dr Marcos Martins Alexandrino da Silva (orientador) - IME-USP

- Prof Dr Claudio Gorodski - IME-USP

- Prof Dr Luiz Amancio Machado de Sousa Junior - UNIRIO 



\section{Agradecimentos}

Ao professor Marcos Alexandrino pela acolhida em São Paulo; por sua douta orientação; pelo tempo que a mim dedicou; por inestimáveis discussões; e pela valiosa contribuição no plano pessoal, meus agradecimentos e minha sincera admiração.

Em meus anos na universidade, outros professores foram de estimada influência. Neste sentido, agradeço a Lorenzo Díaz, Julio Rebelo, Derek Hacon, Henri Anciaux, Alexandre Street e Ronald Shellard na PUC-Rio; Miguel Jorge no Colégio Santo Inácio, que, por motivos diversos, foram suficientemente impactantes durante minha formação.

Agradeço também aos professores Claudio Gorodski e Luiz Amâncio de Sousa por aceitarem fazer parte da banca.

Agradeço a minha avó Clandyra e a meus avós Jorge e Iá (em memória), que se esforçaram em tornar tudo mais fácil para mim.

Sou grato a Claudia, minha mãe, cuja influência positiva em minha vida não posso pôr de forma satisfatória em palavras. Por infundir-me confiança necessária para realizar sonhos, je te remercie infiniment.

No âmbito deste trabalho e da minha estadia em São Paulo, é justo destacar três entidades: ESPN Brasil, TV Justiça e Café Suplicy Jardins.

Por fim, agradeço ao CNPq pela bolsa concedida. 



\section{Resumo}

O objetivo central desta dissertação é apresentar as folheações infinitesimalmente polares, fornecendo uma demonstração para o teorema que as caracteriza. Seguimos a abordagem original encontrada em Lytchak e Thorbergsson [25], de 2010.

Diretamente da definição e do teorema principal obtem-se dois exemplos: folheações polares e folheações riemannianas singulares de codimensão $1 \mathrm{ou}$ 2. Dedicamos especial atenção a um terceiro exemplo: folheações sem pontos horizontalmente conjugados. A demonstração deste resultado utiliza resultados obtidos anteriormente pelos mesmos autores em 2007, em Lytchak e Thorbergsson [24].

Abordamos também, brevemente, as implicações do teorema caracterizador (que é um resultado local) sobre o quociente global de uma folheação infinitesimalmente polar.

Variedades com folheações infinitesimalmente polares podem ser encaradas como um objeto que apresenta aspectos clássicos do teorema do toro maximal para grupos de Lie compactos, em um contexto mais amplo.

Palavras-chave: folheação riemanniana singular; folheação infinitesimalmente polar; explosão de curvatura; pontos horizontalmente conjugados; espaço quociente de uma folheação; mudança de métrica. 



\section{Abstract}

The present work aims at introducing infinitesimally polar foliations - as defined by Lytchak and Thorbergsson [25] - providing a proof for the classification theorem.

Polar foliations and low codimension singular riemannian foliations are two immediate examples. A third example is given by foliations without horizontally conjugate points. The proof of this assertion relies on previous results established by the same authors in [24].

The classification theorem for infinitesimally polar foliations is a local result; we also derive from it some global consequences on the quotient space of such foliations.

Infinitesimally polar foliations may be regarded as a generalised setting where one can find characteristic features from the maximal torus theorem for compact Lie groups.

Keywords: singular Riemannian foliation; infinitesimally polar foliation; curvature explosion; horizontally conjugate points; quotient space of a foliation; change of metric. 



\section{Sumário}

Agradecimentos i

Resumo iii

Abstract $\quad$ V

$\begin{array}{ll}\text { Introdução } & 1\end{array}$

1 Preliminares $\quad 3$

1.1 Curvatura . . . . . . . . . . . . . . . . 3

1.2 Geodésicas . . . . . . . . . . . . . . . 6

1.3 Campos de Jacobi . . . . . . . . . . . . . . . . . . . . . 7

1.4 Isometrias . . . . . . . . . . . . . . . . . . . . 10

1.5 Fórmula de O'Neill . . . . . . . . . . . . . . . . . . . . 12

1.6 Métricas homotéticas . . . . . . . . . . . . . . . . . . . 14

1.7 Subvariedades isoparamétricas . . . . . . . . . . . 15

2 Fatos sobre folheações $\quad 19$

2.1 Ações . . . . . . . . . . . . . . . . . . . . . . . . . . . . . . . . . 19

2.2 O exemplo clássico de ação polar . . . . . . . . . . . . 23

2.3 Folheações singulares . . . . . . . . . . . . . . . . . . 26

2.4 Quocientes e orbifolds . . . . . . . . . . . . . . . . 29

2.5 Vizinhança tubular . . . . . . . . . . . . . . . . . . . . . . . . . . . 34

2.6 Linearização . . . . . . . . . . . . . . . . . . . . . . . . . . 36

2.7 Uma mudança de métrica local . . . . . . . . . . . . . 37

2.8 Folheações polares e isoparamétricas . . . . . . . . . . . . 40

2.9 Reconstruindo folheações . . . . . . . . . . . . . . . . . 43

3 Infinitesimalmente polar $\quad 47$

3.1 Resultado principal . . . . . . . . . . . . . . . . . . . . 47

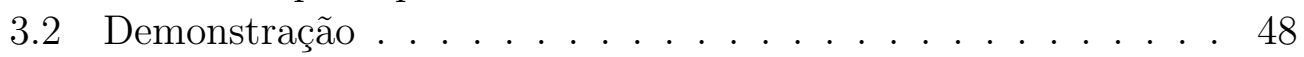

3.3 Dois exemplos . . . . . . . . . . . . . . . . . . . . . 51

vii 
3.4 Completude variacional . . . . . . . . . . . . . . . . . 52

3.5 Caso variacionalmente completo . . . . . . . . . . . . 58

3.6 A estrutura de $M / \mathcal{F} \ldots \ldots$. . . . . . . . . . . . . 62

$\begin{array}{ll}\text { Referências } & 67\end{array}$

$\begin{array}{ll}\text { Índice remissivo } & 71\end{array}$ 


\section{Introdução}

Uma folheação é riemanniana se toda geodésica que é perpendicular a uma folha em um ponto permanece perpendicular a todas as folhas que encontra. Uma folheação singular (seção 2.3) admite folhas de dimensões diferentes. A referência tradicional na área é Molino [27].

Folheações polares (seção 2.8) são folheações riemannianas, singulares e com seções - foram introduzidas em Alexandrino [2] como uma generalização natural para ações polares (seção 2.1). Um fato notável desta classe é que, restrita a um slice, a folheação continua polar e é difeomorfa a uma folheação polar (isoparamétrica) em um espaço euclideano (seção 3.3). Este resultado, conhecido como teorema do slice para folheações polares foi estabelecido em Alexandrino [2]; uma demonstração mais detalhada pode ser encontrada em Piñeros [32].

Por outro lado, folheações polares são também uma generalização natural para famílias isoparamétricas (seção 2.8). Estas famílias constituem um assunto clássico de geometria, sendo, até hoje, um tema de pesquisa em aberto na esfera, por exemplo. Subvariedades isoparamétricas (seção 1.7) em espaços euclideanos, além da rica geometria, possuem objetos algébricos associados. Uma referência usual neste assunto é Palais e Terng [30].

Outra caracterização de polaridade, em Alexandrino [3], é dada pela integrabilidade da distribuição horizontal na parte regular (seção 2.8). As múltiplas abordagens para folheações polares serão fundamentais para demonstração e aplicações do teorema principal 3.5.

Folheações infinitesimalmente polares (seção 3.1) são as que se comportam, em um slice, de maneira similar a polares. Esta classe foi introduzida e caracterizada em Lytchak e Thorbergsson [25]. Entre os exemplos conhecidos estão, claro, as folheações polares (seção 3.3), folheações com codimensão baixa (seção 3.3) e folheações sem pontos horizontalmente conjugados, isto é, ações variacionalmente completas (seção 3.5).

O fato digno de nota é que polaridade infinitesimal fica caracterizada pela estrutura do quociente local ou pelo comportamento de sua curvatura (seção 3.1). O resultado que caracteriza estas folheações (teorema 3.5) diz, em uma 


\section{Folheações infinitesimalmente polares}

primeira abordagem, que são equivalentes:

(i) não-explosão de curvatura no quociente;

(ii) quociente local é bom orbifold;

(iii) polaridade infinitesimal.

O objetivo desta dissertação é apresentar, demonstrar e obter algumas consequências para o teorema 3.5.

O texto está divido em três capítulos.

No capítulo 1, lembramos resultados básicos de geometria riemanniana e fixamos a notação a ser usada ao longo do texto. Além disso, alguns tópicos menos usuais (que tipicamente não são apresentados em um primeiro curso de geometria riemanniana) são relembrados por serem necessários para a demonstração ou para as aplicações do teorema 3.5.

No capítulo 2, reunimos uma breve síntese da teoria de folheações singulares, onde as propriedade relevantes para entender e demonstrar o teorema 3.5 e suas aplicações são apresentadas. Lembramos algumas propriedades de grupos de Lie compactos (teorema do toro maximal) que servem de modelo para grande parte do discutido nesta dissertação.

No capítulo 3, é enunciado o teorema principal 3.5 e feita a sua demonstração. Três seções deste capítulo são dedicadas aos exemplos. Uma última seção discute as implicações do teorema - que é um resultado local - na estrutura do quociente global de folheações.

O leitor familiar com folheações riemannianas singulares (em particular, folheações polares) pode avançar diretamente ao capítulo 3. Visando esta conveniência, discutimos ações variacionalmente completas e folheações associadas na seção 3.4 . 


\section{Capítulo 1}

\section{Preliminares}

Este capítulo é composto, em sua maior parte, por recordação de resultados bem conhecidos de geometria riemanniana. Os fatos são aqui apresentados (sem demonstração) com objetivo de serem utilizados posteriormente - em especial algumas formas de se comparar curvatura secional. Também fixamos a notação a ser usada por todo o texto.

Como referências para o material abordado nas seções 1.1 a 1.6, indicamos Carmo [13], Kolar e outros [23], Petersen [31] ou Chavel [15]. Vamos seguir a convenção de sinais de Carmo [13].

\subsection{Curvatura}

Seja $(M, g)$ uma variedade riemanniana e $T M \rightarrow M$ o seu fibrado tangente. Chamaremos $T_{p} M$ o espaço tangente à $M$ no ponto $p$ e $\Gamma(T M)=\Xi(M)$ o espaço das seções suaves (campos de vetores tangentes em $M$ ) com colchete de Lie $[\because \cdot]$.

Uma função $f: M \rightarrow \mathbb{R}$ tem associada canônicamente uma 1-forma $d f$ sobre $M$ que não depende da métrica $g$. Entender derivadas direcionais de $f$, entretanto, está relacionado a uma forma de derivar campos de vetores. Esta operação necessita de um objeto geométrico que conecte espaços tangentes, no sentido de reduzir o problema rumo ao caso conhecido de funções de $M$ que tomam valores em um mesmo espaço vetorial. Uma conexão afim em $M$ é uma operação sobre $\Xi(M)$

$$
(X, Y) \mapsto \nabla_{X} Y
$$

que é tensorial em $X$ e é uma derivação em $Y$. Neste sentido, podemos entender

$$
\nabla: T M \times \Xi(M) \rightarrow T M
$$




\section{Folheações infinitesimalmente polares}

tal que se $\xi=(p, v) \in T M$ e $Y \in \Xi(M)$, então $\nabla_{\xi} Y \in T_{p} M$; e $\nabla Y$ é uma aplicação suave $T M \rightarrow T M$, isto é,

$$
(\nabla Y)_{p}: T_{p} M \rightarrow T_{p} M
$$

é suave para todo $p \in M$. Quando não há dúvida sobre o ponto base se escreve também $\nabla_{v} Y$. Se $\nabla Y \equiv 0$ dizemos que o campo $Y$ é paralelo (ou $\nabla$-paralelo).

Olhando $\nabla$ em coordenadas é fácil ver que existem infinitas conexões afins sobre uma variedade. É desejável, porém, para se realizar cálculos em $M$, que uma conexão afim apresente mais duas propriedades: seja simétrica $\left(\nabla_{X} Y-\right.$ $\left.\nabla_{Y} X-[X, Y]=0\right)$; e compatível com a métrica $\left(X g(Y, Z)-g\left(\nabla_{X} Y, Z\right)-\right.$ $\left.g\left(Y, \nabla_{X} Z\right)=0\right)$, neste caso, sendo chamada de conexão de Riemann. O teorema de Levi-Civita abaixo é o resultado fundamental para que se possa estudar geometria em uma variedade, garantindo a existência e a unicidade da conexão, além de uma fórmula explícita.

Teorema 1.1. Existe uma única conexão de Riemann $\nabla$ sobre $(M, g)$ determinada pela métrica através da fórmula de Koszul:

$$
\begin{aligned}
2 g\left(\nabla_{X} Y, Z\right)=X \cdot g(Y, Z) & +Y \cdot g(X, Z)-Z \cdot g(X, Y) \\
& -g([Y, X], Z)-g([Y, Z], X)-g([X, Z], Y) .
\end{aligned}
$$

É interessante lembrar que $\left(\nabla_{X} Y\right)_{p}=\nabla_{X_{p}} Y$ só depende do valor de $X$ em $p$ e do valor de $Y$ em uma curva suave $\alpha:(-\varepsilon,+\varepsilon) \rightarrow M$ com $\alpha(0)=p$ e $\alpha^{\prime}(0)=X_{p}$.

O tensor de curvatura de Riemann $R$ é dado em termos da conexão por

$$
R(X, Y) Z:=\nabla_{Y} \nabla_{X} Z-\nabla_{X} \nabla_{Y} Z+\nabla_{[X, Y]} Z .
$$

Usaremos também a notação

$$
R(X, Y, Z, W):=g(R(X, Y) Z, W) .
$$

O tensor de curvatura pode ser um objeto complicado. Introduz-se objetos mais simples e tratáveis que apesar de, por definição, guardarem menos informação, permitem obter resultados interessantes. Destacamos aqui o conceito de curvatura secional.

A escolha de dois vetores tangentes linearmente independentes $X$ e $Y$ em $p \in M$ é equivalente a escolha de um 2-plano $\sigma_{p}$ em $T_{p} M$. A curvatura secional de $M$ com respeito a $\sigma_{p}$ é

$$
k(X, Y)=k\left(\sigma_{p}\right):=\frac{R(X, Y, X, Y)}{g(X, X) \cdot g(Y, Y)-g(X, Y) \cdot g(X, Y)} .
$$




\subsection{Curvatura}

Observamos que o denominador da expressão é o quadrado da área de

$$
\{t X+s Y: 0 \leqslant t, s \leqslant 1\} \subseteq T_{p} M
$$

e que $k\left(\sigma_{p}\right)$ não depende da escolha de $X$ e $Y$.

É possível definir naturalmente uma estrutura diferenciável em $G_{2}(M)$, a coleção de todos os 2-planos tangentes à $M$. Desta forma,

$$
k: G_{2}(M) \rightarrow \mathbb{R}
$$

está bem-definida e é uma operação suave.

Um fato relevante é que o conhecimento de $k$ para todos os planos tangentes contém toda a informação sobre a curvatura da variedade, no sentido em que é possível se recompor o tensor $R$ a partir desses dados.

Proposição 1.5. O tensor de curvatura $R$ em um ponto $p$ está unicamente determinado pela curvatura secional de todos os 2-planos em $T_{p} M$.

Um espaço com curvatura secional constante igual a zero será chamado flat. Neste sentido, uma das informações que a curvatura guarda é o quanto o espaço deixa de ser euclideano. Uma variedade flat é localmente isométrica a um espaço euclideano.

Para interpretações do conceito de curvatura e objetos derivados em geometria riemanniana, recomendamos Berger [7].

Considere uma subvariedade $N$ de $M$. Uma métrica $g$ em $M$ fornece noção de ortogonalidade e permite decompor

$$
T M=T N \oplus \nu N
$$

onde $\nu N$ é o fibrado normal de $N$, isto é, $\nu_{p} N$ é complemento ortogonal (em $\left.T_{p} M\right)$ de $T_{p} N$ em relação à métrica $g$.

É possível derivar os campos em $N$ seguindo essa decomposição; escrevemos, para todos $X, Y \in \Xi(N)$, a decomposição única

$$
\nabla_{X} Y=\left(\nabla_{X} Y\right)^{\top}+\left(\nabla_{X} Y\right)^{\perp}
$$

onde $\nabla$ é a conexão de $M$ e $\left(\nabla_{X} Y\right)^{\top}=: \nabla_{X}^{N} Y$ é exatamente a conexão riemanniana em $N$ dada pela métrica induzida por $g$.

Temos que $\operatorname{II}(X, Y):=\left(\nabla_{X} Y\right)^{\perp}$ é a segunda forma fundamental de $N$. Se $\mathrm{II} \equiv 0$ diremos que $N$ é uma subvariedade totalmente geodésica (na próxima seção será dado sentido ao nome).

Se $\xi$ é um campo normal em $N$ define-se o operador-forma de $N$ na direção $\xi$ como

$$
W_{\xi} X:=-\left(\nabla_{X} \xi\right)^{\top}
$$




\section{Folheações infinitesimalmente polares}

Vale que $W_{\xi}$ é auto-adjunto em cada espaço tangente à $N$ e logo diagonalizável. Os autovalores de $W_{\xi}$ são as curvaturas principais de $N$ na direção $\xi$.

\subsection{Geodésicas}

A métrica $g$ permite que se defina em $M$ naturalmente a distância dist $(p, q)$ entre dois pontos $p, q \in M$ quaisquer como o comprimento do menor caminho entres eles, onde o comprimento de uma curva $\gamma:[a, b] \rightarrow M$ diferenciávelpor-partes é

$$
\int_{a}^{b} \sqrt{g\left(\gamma^{\prime}(t), \gamma^{\prime}(t)\right)} d t
$$

Desta forma, ( $M$, dist) é um espaço métrico.

Seja $\gamma: I \rightarrow M$ suave. Existe um fibrado $\gamma^{*} T M \rightarrow I \subseteq \mathbb{R}$ cujas seções são os campos suaves ao longo de $\gamma$. Este fibrado tem uma conexão $D_{t}$ dada pelo pullback da conexão $\nabla$ de $M$. O operador

$$
D_{t}: \Gamma\left(\gamma^{*} T M\right) \rightarrow \Gamma\left(\gamma^{*} T M\right)
$$

é único para cada $\gamma$ e é uma derivação, chamado derivada covariante ao longo de $\gamma$. Se $X$ é um campo sobre $\gamma$ e $\tilde{X} \in \Xi(M)$ é tal que $\left.\tilde{X}\right|_{\gamma}=X$, então

$$
D_{t} X=\nabla_{\gamma^{\prime}} \tilde{X}
$$

Diremos que uma curva $\gamma:[a, b] \rightarrow M$ é uma geodésica quando

$$
D_{t} \gamma^{\prime} \equiv 0
$$

A equação (1.8) se lê como $\gamma^{\prime}$ ser paralelo ao longo da curva $\gamma$. Em geral, um campo $X$ é paralelo quando $\nabla_{v} X=0$ para todo vetor tangente $v$ em $M$, ou seja, quando é paralelo ao longo de todas as curvas em $M$.

Se $v \in T_{p} M$ é um vetor tangente à $M$ em $p$ e $\alpha:[0,1] \rightarrow M$ é uma curva com $\alpha(0)=p$, existe um único campo $X$ paralelo ao longo de $\alpha$ que verifica $X_{p}=v$. Diz-se que $X$ é o transporte paralelo de $v$ ao longo de $\alpha$.

É possível ver que a segunda forma fundamental II é simétrica e daí decorre, em vista da equação (1.7), que uma subvariedade $N$ de $M$ é totalmente geodésica se, e somente se, toda geodésica de $N$ (na métrica induzida) é geodésica de $M$.

Podemos definir um fluxo geodésico no fibrado tangente. Um resultado clássico de equações diferenciais ordinárias nos dá o seguinte. 


\subsection{Campos de Jacobi}

Proposição 1.9. Para todo $\xi=(p, v) \in T M$ existe uma única geodésica $\gamma_{\xi}: I_{\xi} \rightarrow M$ tal que

$$
\left\{\begin{array}{l}
\gamma_{\xi}(0)=p \\
\gamma_{\xi}^{\prime}(0)=v
\end{array}\right.
$$

onde $I_{\xi}$ é um intervalo maximal na reta contendo a origem.

Seja

$$
V:=\left\{\xi \in T M: 1 \in I_{\xi}\right\}
$$

Definimos

$$
\begin{aligned}
\exp : V & \rightarrow M \\
\xi & \mapsto \gamma_{\xi}(1) .
\end{aligned}
$$

Para todo $p \in M$, seja $V_{p}=T_{p} M \cap V$ e $\exp _{p}=\left.\exp \right|_{V_{p}}$. A aplicação assim definida é chamada de exponencial.

É um fato curioso que $V_{p}=T_{p} M$ para algum $p \in M$ se, e somente se, $V=$ $T M$. Quando isso acontece dizemos que $M$ é uma variedade geodesicamente completa.

Pode-se mostrar que

$$
\gamma_{\xi}(t)=\exp (t \xi)
$$

onde a expressão faz sentido, logo uma variedade é geodesicamente completa se as geodésicas de $M$ estão definidas em toda a reta $\mathbb{R}$.

Notamos ainda que as duas noções de completude comentadas são equivalentes. O próximo teorema é parte de uma série de equivalências que compõem o teorema de Hopf e Rinow.

Teorema 1.10. Temos que $(M, g)$ é completa (geodesicamente) se, e somente se, ( $M$, dist) é completa (como espaço métrico).

Uma forma espacial é uma variedade riemanniana completa com curvatura secional constante. Os exemplos elementares são esferas e espaços euclideanos. Usaremos a notação $M^{n}(k)$ para a forma espacial de curvatura $k$ e dimensão $n$. Em particular, $M^{n}(1)=\mathbb{S}^{n}$.

\subsection{Campos de Jacobi}

Seja $\gamma: I \rightarrow M$ uma geodésica, uma variação por geodésicas de $\gamma$ é uma família de geodésicas $\gamma_{s}: I \rightarrow M$, suave em $s \in(-\varepsilon,+\varepsilon)$ tal que $\gamma_{0}=\gamma$. Uma tal família induz um campo $J$ em $\gamma$ dado por

$$
J(t):=\left.\frac{d}{d s} \gamma_{s}(t)\right|_{s=0}
$$




\section{Folheações infinitesimalmente polares}

chamado de variação infinitesimal.

Mostra-se que uma variação infinitesimal deve satisfazer a equação de Jacobi:

$$
D_{t} D_{t} J+R\left(\gamma^{\prime}, J\right) \gamma^{\prime}=0
$$

onde $D_{t}$ é a derivada covariante ao longo de $\gamma$. Um campo ao longo de $\gamma$ que verifica a equação (1.12) é um campo de Jacobi.

Variações por geodésicas e campos de Jacobi estão completamente determinados dadas duas condições iniciais, daí segue o seguinte.

Proposição 1.13. Um campo J sobre uma geodésica $\gamma$ é um campo de Jacobi se, e somente se, é variação infinitesimal de uma variação por geodésicas de $\gamma$.

Um campo de Killing em $(M, g)$ é um campo vetorial cujo fluxo é uma isometria, ou seja,

$$
L_{X} g=0,
$$

onde $L$ é a derivada de Lie.

Diz-se que um campo de Killing $X$ é um gerador infinitesimal de isometrias no sentido em que tomando-se um subconjunto $A \subseteq M$ e o deslocando (pontualmente) seguindo o fluxo de $X$ as distâncias em $A$ não se alteram.

Um campo $X$ é de Killing se, e somente se, verifica, para todos $X, Y, Z \in$ $\Xi(M)$, a equação de Killing:

$$
g\left(\nabla_{Y} X, Z\right)+g\left(\nabla_{Z} X, Y\right)=0
$$

Lema 1.15. A restrição de um campo de Killing a uma geodésica é sempre um campo de Jacobi.

Demonstração. Seja $X$ um campo de Killing, $\varphi_{s}$ seu fluxo e $\gamma$ uma geodésica. Então $X$ restrito a $\gamma$ é a variação infinitesimal de $\gamma_{s}:=\varphi_{s} \circ \gamma$.

Dois pontos $p$ e $q$ em $M$ são conjugados (por $\gamma$ ) se existe uma geodésica $\gamma:[0,1] \rightarrow M$ com $\gamma(0)=p, \gamma(1)=q$ e um campo de Jacobi não-nulo $J$ sobre $\gamma$ tal que

$$
J(0)=0=J(1) .
$$

O conjunto dos campos de Jacobi ao longo de $\gamma$ que se anulam nas extremidades é um espaço vetorial $\mathcal{J}_{\gamma}$ cuja dimensão é a multiplicidade dos pontos conjugados. Se $\mathcal{J}_{\gamma}$ é trivial os campos de Jacobi sobre $\gamma$ estão determinados no sentido da próxima proposição. 


\subsection{Campos de Jacobi}

Proposição 1.16. Considere $p$ e q em uma variedade $M$ ligados por uma geodésica $\gamma$ como acima e tais que não sejam conjugados por $\gamma$. Então, dados $v_{0} \in T_{p} M$ e $v_{1} \in T_{q} M$, existe um único campo de Jacobi $J$ sobre $\gamma$ tal que $J(0)=v_{0}$ e $J(1)=v_{1}$.

Temos que $\mathcal{J}_{\gamma}$ é subespaço vetorial de $\mathcal{V}_{\gamma}$, o conjunto de todos os campos ao longo de $\gamma$ que se anulam nas extremidades. O conceito de ponto conjugado se relaciona com teoria de Morse através de uma forma bilinear $I \mathrm{em}$ $\mathcal{V}_{\gamma}$ oriunda de técnicas de variação de energia:

$$
\begin{aligned}
I(X, Y):=\int_{0}^{1} g\left(D_{t} X(t), D_{t} Y(t)\right) & \\
& \quad-R\left(\gamma^{\prime}(t), D_{t} X(t), \gamma^{\prime}(t), D_{t} Y(t)\right) d t .
\end{aligned}
$$

Os elementos $X$ de $\mathcal{V}_{\gamma}$ tais que $I(X, \cdot) \equiv 0$ são precisamente os campos de Jacobi $\mathcal{J}_{\gamma}$. Segue que $p$ e $q$ são conjugados por $\gamma$ se $I$ for degenerada. A dimensão de ker $I$ é a multiplicidade dos pontos conjugados.

O índice de $I$ é a dimensão de um subespaço maximal de $\mathcal{V}_{\gamma}$ onde $I$ é negativa-definida. Vale que é o índice é sempre finito.

Teorema 1.18. O indice de I é igual ao número de pontos em $\gamma$ conjugados a $\gamma(0)$ contados com suas multiplicidades.

O resultado acima é chamado teorema do indice de Morse. Segue daí que o conjunto de pontos conjugados em uma geodésica é discreto. Nesta mesma abordagem, conclui-se que um segmento de geodésica é minimizador de distâncias entre curvas próximas se, e somente se, não possui pontos conjugados.

A noção de ponto conjugado pode ser generalizada no sentido de substituir um dos pontos por uma subvariedade. Este conceito está ligado a um tipo especial de campos de Jacobi.

Seja $L$ uma subvariedade imersa de $M$. Seja $\gamma$ uma geodésica perpendicular à $L$, isto é, tal que $\gamma(0) \in L$ e $\gamma^{\prime}(0) \in \nu_{\gamma(0)} L$. Um campo $L$-Jacobi ao longo de $\gamma$ é um campo de Jacobi que é variação infinitesimal de uma variação por geodésicas todas ortogonais à $L$.

Seja $\nu L$ o fibrado normal de $L$ e

$$
\eta:=\exp ^{\perp}: \nu L \rightarrow M
$$

a restrição da aplicação exponencial. Se $d \eta$ é singular em $\xi$, então chamamos $\xi$ de vetor focal e $\exp ^{\perp}(\xi)$ de ponto focal de $L$.

A multiplicidade de um ponto focal é a dimensão do núcleo de

$$
d \eta_{v}: T_{v} \nu L \rightarrow T_{\eta(v)} M .
$$




\section{Folheações infinitesimalmente polares}

Definição 1.19. Se $\xi \in \nu L$ é um campo normal em $L$, definimos a aplicação ponto-final na direção $\xi$ como

$$
\begin{aligned}
\eta_{\xi}: L & \rightarrow M \\
x & \mapsto \eta_{\xi}(x)=\exp _{x}^{\perp}\left(\xi_{x}\right) .
\end{aligned}
$$

Proposição 1.20. Um ponto $\gamma(t)$ em uma geodésica perpendicular à $L$ é um ponto focal de $L$ se, e somente se, existe um campo L-Jacobi $J$ não-nulo em $\gamma$ tal que $J(t)=0$.

Lembramos que um campos de Jacobi $J$ sobre uma geodésica $[a, b] \rightarrow$ $M$ está completamente determinado pelas duas condições iniciais $J(a)$ e $\left(D_{t} J\right)(a)$. Campos $L$-Jacobi também estão relacionados a duas condições iniciais.

Seja $\gamma$ geodésica em $M$ ortogonal a uma subvariedade $L$ em $p=\gamma(0)$, assim, vide equação (1.6), $W_{\gamma^{\prime}(0)}: T_{p} L \rightarrow T_{p} L$ está bem-definido.

Proposição 1.21. Seja J campo de Jacobi sobre $\gamma$. Então J é L-Jacobi se, e somente se,

$$
\left\{\begin{array}{lll}
J(0) & \in T_{p} L \\
\left(D_{t} J\right)(0)-W_{\gamma^{\prime}(0)}(J(0)) & \in \nu_{p} L
\end{array}\right.
$$

Uma demonstração para esta proposição pode ser encontrada em Carmo [13], capítulo 10.

\subsection{Isometrias}

Considere variedades riemannianas $(M, g)$ e $\left(M^{\prime}, g^{\prime}\right)$. Uma aplicação $\varphi$ : $M \rightarrow M^{\prime}$ é uma isometria local (riemanniana) se

$$
g=\varphi^{*} g^{\prime}
$$

isto é, para todos $x \in M, X, Y \in T_{x} M$, temos

$$
g_{x}(X, Y)=g_{\varphi(x)}^{\prime}\left(d \varphi_{x}(X), d \varphi_{x}(Y)\right) .
$$

Se, além disso, $\varphi$ for um difeomorfismo dizemos que é uma isometria (global).

Isometrias fornecem uma noção de igualdade geométrica entre variedades riemannianas, no sentido em que preservam as estruturas relevantes. Seja $\varphi:(M, g) \rightarrow\left(M^{\prime}, g^{\prime}\right)$ isometria e considere dist e dist', $\nabla$ e $\nabla^{\prime}, R$ e $R^{\prime}, k$ e $k^{\prime}$ as funções distância, conexões, tensores de curvatura, curvaturas secionais de $(M, g)$ e $\left(M^{\prime}, g^{\prime}\right)$, respectivamente. 


\subsection{Isometrias}

Temos, para todos $p, q \in M$,

$$
\operatorname{dist}^{\prime}(\varphi(p), \varphi(q))=\operatorname{dist}(p, q),
$$

e, para todos $X, Y \in \Xi(M)$,

$$
d \varphi\left(\nabla_{X} Y\right)=\nabla_{d \varphi X}^{\prime} d \varphi Y
$$

Da equação (1.22) fica claro que os tensores de curvatura também são preservados:

$$
R^{\prime}=\varphi^{*} R
$$

Em particular, um objeto de nosso especial interesse neste texto, a curvatura secional.

Proposição 1.23. Na notação acima,

$$
k_{p}(X, Y)=k_{\varphi(p)}^{\prime}(d \varphi X, d \varphi Y) .
$$

Note que a conexão é um objeto intrínseco (completamente determinado via equação (1.2) pela métrica) e logo a condição $D_{t} \gamma^{\prime}=0$ para uma dada curva também. Assim, vemos que isometrias levam geodésicas em geodésicas.

Podemos, mais precisamente, afirmar que

$$
\varphi \circ \exp _{p}(X)=\exp _{\varphi(p)}\left(d \varphi_{p} X\right)
$$

Observação 1.24. Seja $(M, g)$ variedade riemanniana e $\varphi$ um difeomorfismo de um subconjunto aberto (ou uma subvariedade) $U \subseteq M$ para $V:=\varphi(U) \subseteq M$. Usando a definição de isometria, se consideramos a métrica $g=\left.g\right|_{V}$ em $V$, é possível dotar $U$ com uma métrica $\tilde{g}$ tal que

$$
\varphi:(U, \tilde{g}) \rightarrow(V, g)
$$

seja uma isometria, a saber,

$$
\tilde{g}:=\varphi^{*} g .
$$

E se $\tilde{\nabla}$ e $\nabla$ são as conexões em $(U, \tilde{g})$ e $(V, g)$, respectivamente, temos

$$
d \varphi\left(\tilde{\nabla}_{X} Y\right)=\nabla_{d \varphi X} d \varphi Y .
$$

Uma imersão injetiva $\varphi:(M, g) \rightarrow\left(M^{\prime}, g^{\prime}\right)$ é um mergulho isométrico se preserva a métrica, ou seja, $g=\varphi^{*} g^{\prime}$. Observamos que se $f$ é um mergulho suave que preserva o comprimento de curvas, então $f$ é um mergulho isométrico (e vice versa). Convém observar que este fato está relacionado 


\section{Folheações infinitesimalmente polares}

ao notável teorema de Nash que afirma ser possível mergulhar isometricamente qualquer variedade riemanniana em um espaço euclideano $\mathbb{R}^{m}$, para $m$ suficientemente grande.

Finalizamos esta seção com o seguinte resultado - uma recíproca parcial da propriedade (vista acima) que distâncias não são alteradas por isometrias -, conhecido como teorema de Myers e Steenrod, que fornece um critério poderoso para encontrar isometrias.

Teorema 1.25. Sejam ( $M$, dist) e ( $M^{\prime}$, dist' ${ }^{\prime}$ variedades riemannianas e seja $\varphi: M \rightarrow M^{\prime}$ uma aplicação sobrejetora que preserva distâncias, isto é, tal que

$$
\operatorname{dist}^{\prime}(\varphi(p), \varphi(q))=\operatorname{dist}(p, q)
$$

para todo $p, q \in M$. Então $\varphi$ é uma isometria.

Observe que o resultado acima não exige que a aplicação $\varphi$ seja contínua em princípio, mas a conclusão implica na sua suavidade. Um roteiro para a demonstraçao pode ser encontrado, por exemplo, em Chavel [15], capítulo IV.

\subsection{Fórmula de O’Neill}

Nesta seção, são relembrados rapidamente alguns resultados visando enunciar a fórmula de O'Neill, que fornece uma importante ferramenta de comparação de curvaturas secionais no caso de submersões riemannianas. Um tratamento com mais detalhes pode ser encontrado, por exemplo, em O'Neill [29] ou nos exercícios do capítulo 8 de Carmo [13].

Uma aplicação $\pi: M \rightarrow B$ entre variedades riemannianas $(M, g)$ e $\left(B, g^{\prime}\right)$ é uma submersão se

$$
d \pi_{p}: T_{p} M \rightarrow T_{\pi(p)} B
$$

é sobrejetora para todo $p \in M$. Vale que $\pi^{-1}(b) \subseteq M$ é uma subvariedade mergulhada (para todo $b$ na imagem) chamada fibra sobre $b$. Em geral, chama-se $B$ de base e $M$ de espaço total.

Seja $M_{p}:=\pi^{-1}(\pi(p))$, definimos a distribuição

$$
V_{p}:=\operatorname{ker}\left(d \pi_{p}\right)=T_{p} M_{p}
$$

que é o espaço vertical (da submersão) no ponto $p$, isto é, o espaço tangente à fibra que passa naquele ponto. Analogamente, temos uma distribuição horizontal

$$
H_{p}:=V_{p}^{\perp}
$$




\subsection{Fórmula de O’Neill}

É claro que $T_{p} M$ é soma direta dos espaços horizontal e vertical ( $\pi$ ser submersão garante as distribuições suaves e não-degeneradas).

Um campo $X$ em $M$ é dito horizontal se $X_{p} \in H_{p}$ para todo $p$ e vertical se $X_{p} \in V_{p}$ para todo $p$. Para um campo $Y$ qualquer, escrevemos $Y=Y^{h}+Y^{v}$ sua decomposição única em partes horizontal e vertical.

Uma submersão é dita riemanniana se respeita as métricas no sentido em que, para quaisquer $X, Y$ horizontais em $M$, se tem

$$
g(X, Y)=g^{\prime}(d \pi X, d \pi Y)
$$

ou seja, o isomorfismo

$$
d \pi_{p}: H_{p} \rightarrow T_{\pi(p)} B
$$

é uma isometria.

Para cada campo $Z$ em $B$, há um único campo horizontal $\pi$-relacionado $\tilde{Z}$ em $M$, isto é,

$$
d \pi_{p}\left(\tilde{Z}_{p}\right)=Z_{\pi(p)} .
$$

O campo $\tilde{Z}$ é chamado levantamento horizontal de $Z$.

Curvas em $B$ também podem ser levantadas. Seja $\alpha:[0,1] \rightarrow B$ curva suave e $x \in \pi^{-1}(\alpha(0))$, existe única curva $\tilde{\alpha}:[0,1] \rightarrow M$ tal que

$$
\pi \circ \tilde{\alpha}=\alpha
$$

$\operatorname{com} \tilde{\alpha}(0)=x$ e $\tilde{\alpha}^{\prime}(t) \in H_{\tilde{\alpha}(t)}$. A curva $\tilde{\alpha}$ é chamada também de levantamento horizontal de $\alpha$.

O levantamento horizontal de geodésicas em $B$ nos dá também geodésicas em $M$. Mais precisamente, temos o seguinte.

Lema 1.26. Na situação acima, seja $\gamma: I \rightarrow M$ curva horizontal. Então $\gamma$ é uma geodésica se, e somente se, $\pi \circ \gamma$ for geodésica em $B$.

Considere ainda uma submersão riemanniana $\pi: M \rightarrow B$ com $k_{M}$ e $k_{B}$ as curvaturas secionais de $M$ e $B$. Seguindo a notação desta seção, as curvaturas secionais dos espaços de chegada e total estão relacionadas pela fórmula de O'Neill:

$$
k_{B}(X, Y)=k_{M}(\tilde{X}, \tilde{Y})+\frac{3}{4} \frac{g\left([\tilde{X}, \tilde{Y}]^{v},[\tilde{X}, \tilde{Y}]^{v}\right)}{g(\tilde{X}, \tilde{X}) \cdot g(\tilde{Y}, \tilde{Y})-g(\tilde{X}, \tilde{Y}) \cdot g(\tilde{X}, \tilde{Y})}
$$

que, caso $\tilde{X}$ e $\tilde{Y}$ sejam ortonormais, se reduz para

$$
k_{B}(X, Y)=k_{M}(\tilde{X}, \tilde{Y})+\frac{3}{4}\left\|[\tilde{X}, \tilde{Y}]^{v}\right\|^{2} .
$$




\section{Folheações infinitesimalmente polares}

A equação (1.28) é válida, em particular, quando os levantamentos são fixados a priori, ou seja, quando se fixa vetores ortonormais $\tilde{X}$ e $\tilde{Y}$ em $p \in$ $M$. É claro que $\tilde{X}$ é o levantamento horizontal do vetor $X:=d \pi_{p}(\tilde{X})$ em $\pi(p) \in B$ e toma-se $Y$ de forma análoga.

Note que, em particular, a equação (1.27) implica

$$
k_{B}(X, Y) \geqslant k_{M}(\tilde{X}, \tilde{Y})
$$

isto é, em submersões riemannianas, a base carrega mais curvatura do que o espaço total.

$\mathrm{Na}$ exposição original em O’Neill [29], são definidos dois tensores fundamentais em $M$, sendo um vertical e um horizontal. O tensor horizontal é

$$
A_{X} Y:=\left(\nabla_{X^{h}} Y^{h}\right)^{v}+\left(\nabla_{X^{h}} Y^{v}\right)^{h}
$$

que mede a integrabilidade da distribuição horizontal no sentido seguinte.

Lema 1.30. Se $X$ e $Y$ são horizontais, então $A_{X} Y=\frac{1}{2}[X, Y]^{v}$.

Segue que a distribuição horizontal $H$ em $M$ é integrável (segundo Frobenius) se, e somente se, $A_{X}=-A X \equiv 0$ para todo campo $X$ em $H$.

Finalmente, podemos escrever a equação (1.27) usando $A$. Se $\tilde{X}$ e $\tilde{Y}$ são ortonormais temos:

$$
k_{B}(X, Y)=k_{M}(\tilde{X}, \tilde{Y})+3\left\|A_{\tilde{X}} \tilde{Y}\right\|^{2} .
$$

\subsection{Métricas homotéticas}

Esta seção é composta de alguns cálculos diretos - que serão usados mais tarde nas seções 3.2, 3.4 e 3.5 - descrevendo o que acontece para certas mudanças simples de métrica.

Considere uma variedade riemanniana $(M, g)$ e $\eta$ um número real positivo. Defina uma nova métrica $g^{\eta}$ em $M$ por

$$
g^{\eta}:=\eta^{2} g=g(\eta \cdot, \eta \cdot) .
$$

Seja $\nabla^{\eta}$ a conexão de Riemann associada à nova métrica $g^{\eta}$, temos, da 


\subsection{Subvariedades isoparamétricas}

equação (1.2),

$$
\begin{aligned}
2 g^{\eta}\left(\nabla_{X}^{\eta} Y, Z\right)= & X \cdot g^{\eta}(Y, Z)+Y \cdot g^{\eta}(X, Z)-Z \cdot g^{\eta}(X, Y) \\
& -g^{\eta}([Y, X], Z)-g^{\eta}([Y, Z], X)-g^{\eta}([X, Z], Y) \\
= & X \eta^{2} g(Y, Z)+Y \cdot \eta^{2} g(X, Z)-Z \cdot \eta^{2} g(X, Y) \\
& -\eta^{2} g([Y, X], Z)-\eta^{2} g([Y, Z], X)-\eta^{2} g([X, Z], Y) \\
= & \eta^{2}\left(2 g\left(\nabla_{X} Y, Z\right)\right),
\end{aligned}
$$

e, por outro lado,

$$
2 g^{\eta}\left(\nabla_{X}^{\eta} Y, Z\right)=\eta^{2}\left(2 g\left(\nabla_{X}^{\eta} Y, Z\right)\right),
$$

e logo

$$
\nabla^{\eta}=\nabla
$$

Agora seja $R^{\eta}$ o tensor de curvatura associado com a conexão $\nabla^{\eta}$ acima. É claro, a partir da equação (1.3), que

$$
R^{\eta}=R .
$$

Finalmente, a curvatura secional da métrica $g^{\eta}$ está também relacionada de forma simples àquela da métrica original $g$ : temos

$$
\begin{aligned}
k^{\eta}\left(\sigma_{p}\right) & =\frac{g^{\eta}\left(R^{\eta}(X, Y) X, Y\right)}{g^{\eta}(X, X) \cdot g^{\eta}(Y, Y)-g^{\eta}(X, Y) \cdot g^{\eta}(X, Y)} \\
& =\frac{\eta^{2} g(R(X, Y) X, Y)}{\eta^{2} g(X, X) \cdot \eta^{2} g(Y, Y)-\eta^{2} g(X, Y) \cdot \eta^{2} g(X, Y)} \\
& =\frac{\eta^{2}}{\eta^{4}} k\left(\sigma_{p}\right),
\end{aligned}
$$

ou seja,

$$
k^{\eta}=\frac{1}{\eta^{2}} k .
$$

\subsection{Subvariedades isoparamétricas}

As folheações isoparamétricas serão introduzidas na seção 2.8; são folheações riemannianas com seção e têm um papel importante tanto na demonstração do teorema 3.5 quanto nas aplicações. Veremos que as folhas regulares destas 


\section{Folheações infinitesimalmente polares}

folheações são subvariedades isoparamétricas. Esta seção é uma coleção de fatos sobre subvariedades isoparamétricas que serão necessários na seção 2.8.

Demonstrações para os resultados aqui enunciados podem ser encontradas em, por exemplo, Palais e Terng [30] ou Chamorro [14].

Esta abordagem de famílias isoparamétricas, no sentido de generalizar resultados clássicos, foi introduzida em Terng [36]. Um survey recente pode ser encontrado em Thorbergsson [37].

Uma subvariedade de uma forma espacial $M(k)$ é isoparamétrica se seu fibrado normal é flat e suas curvaturas principais ao longo de qualquer campo vetorial normal paralelo são constantes.

Seja $L$ uma subvariedade isoparamétrica em $M(k)$ e $\xi$ um vetor normal paralelo ao longo de $L$. Defina

$$
L_{\xi}:=\left\{\eta_{\xi}(p): p \in L\right\},
$$

onde $\eta_{\xi}$ é aplicação ponto-final definida na seção 1.3.

Se $L_{\xi}$ tem a dimensão de $L$, dizemos que é uma variedade paralela. Caso contrário, dizemos que é uma variedade focal de $L$.

Proposição 1.36. Uma variedade paralela de uma variedade isoparamétrica é também uma variedade isoparamétrica.

Agora vamos enumerar alguns resultados úteis para espaços euclideanos.

Lema 1.37. Uma subvariedade é isoparamétrica na esfera $\mathbb{S}^{n}$ se, e somente se, é isoparamétrica no espaço $\mathbb{R}^{n+1}$.

Lema 1.38. Uma subvariedade é isoparamétrica em $\mathbb{R}^{n}$ se, e somente se, é isoparamétrica no espaço $\mathbb{R}^{n+1}$.

Proposição 1.39. Uma subvariedade isoparamétrica e completa de um espaço euclideano é compacta se, e somente se, está contida em uma esfera.

Há uma relação estreita entre subvariedades isoparamétricas e certas aplicações.

Definição 1.40. Uma função $f: M \rightarrow \mathbb{R}$ em uma variedade riemanniana $M$ é transnormal se

$$
\|\operatorname{grad} f\|^{2}=b(f),
$$

onde $b$ é uma função real suave. Se, além disso,

$$
\Delta f=a(f),
$$

onde $a$ é uma função real suave e $\Delta f$ é o laplaceano de $f$, dizemos que $f$ é isoparamétrica. 


\subsection{Subvariedades isoparamétricas}

As quantidades $\|\operatorname{grad} f\|^{2}$ e $\Delta f$ são historicamente chamadas de parâmetros diferenciais de $f$. A definição acima significa dizer que funções isoparamétricas são aquelas cujos parâmetros são constantes em seus conjuntos de nível.

Uma subvariedade $L$ de $M$ é uma hiperfície isoparamétrica se é dada localmente como pré-imagem de uma função isoparamétrica. Em formas espaciais, esta definição coincide com a anterior.

Uma hiperfície é regular se é pré-imagem de um valor regular. Se $f$ é transnormal, então, variando $k$ entre valores regulares,

$$
L_{k}:=\left\{f^{-1}(k)\right\}
$$

são hiperfícies paralelas exatamente como aquelas obtidas pela aplicação ponto-final: fixando um valor de $k$ qualquer e tomando $\left(L_{k}\right)_{\xi}$. (As préimagens de valores não-regulares são hiperfícies focais.) Se $f$ for isoparamétrica, então a soma das curvaturas principais é constantes nas hiperfícies regulares ou paralelas.

Observação 1.41. Em Wang [40], é afirmado que as pré-imagens de máximos ou mínimos de funções isoparamétricas em variedades completas são superfícies mínimas.

Assim, podemos entender a definição 1.40 de funções isoparamétricas como uma aplicação que gera famílias de hiperfícies com soma das curvaturas principais constantes. (Em formas espaciais, continua valendo a conclusão mais forte de que as curvaturas principais são constantes.) Ou seja: uma hiperfície é isoparamétrica quando suas hiperfícies paralelas têm curvatura média constante.

Proposição 1.42. Uma hiperfície isoparamétrica no espaço euclideano é um subconjunto aberto de

(i) um hiperplano; ou

(ii) uma esfera; ou

(iii) um cilindro (sobre esfera).

É possível generalizar esta abordagem para definir subvariedade isoparamétricas de codimensão maior em variedades através de aplicações isoparamétricas. Ver, por exemplo, Palais e Terng [30]. 



\section{Capítulo 2}

\section{Fatos sobre folheações}

Neste capítulo, vamos recordar alguns resultados clássicos da teoria de ações e introduzir folheações singulares como contexto natural para generalização destes. O foco é em folheações riemannianas singulares, visando construir o material necessário para entender o enunciado e a demonstração do teorema 3.5. Apesar dos fatos serem apresentados como ferramentas para o caso polaridade infinitesimal, diversos resultados aqui enunciados são teoremas relevantes por si só, fruto de pesquisa recente.

As principais referências para este capítulo são Alexandrino e Bettiol [5], Palais e Terng [30], Duistermaat e Kolk [19], Molino [27], Piñeros [32] e Alexandrino e Toeben [6].

\subsection{Ações}

Esta seção é uma recordação de alguns fatos bem conhecidos sobre ações de grupos de Lie, com ênfase em ações polares.

Um grupo de Lie Gé um grupo que possui estrutura de variedade tal que a operação

$$
\begin{aligned}
G \times G & \rightarrow G \\
(x, y) & \mapsto x y^{-1}
\end{aligned}
$$

é suave (esta condição equivale a suavidade do produto e da inversa). A dimensão de $G$ é a sua dimensão como variedade.

A cada grupo de Lie $G$ está associada uma álgebra de Lie $\mathfrak{g}$ isomorfa ao $T_{e} G$, onde $e$ é a identidade de $G$, com o colchete de Lie como operação (dados dois vetores em $T_{e} G$, toma-se o colchete na origem de campos que os estendam). 


\section{Folheações infinitesimalmente polares}

Exemplos imediatos de grupos de Lie são: os reais $\mathbb{R}$ com operação adição; os complexos não-nulos $\mathbb{C}^{*}$ com operação produto; o grupo de automorfismos $G L(V)$ de um espaço vetorial $V$ (de dimensão finita).

É fácil ver que $\mathbb{S}^{1}$ é um grupo de Lie (com a operação produto de $\mathbb{C}^{*}$ induzida), bem como $\mathbb{T}^{n}:=\mathbb{S}^{1} \times \cdots \times \mathbb{S}^{1}$.

Um grupo de Lie isomorfo (relacionado via homomorfismo suave) ao produto de $n$ cópias de $\mathbb{S}^{1}$ será chamado de $n$-toro - sua álgebra de Lie é $\mathbb{R}^{n}$.

Observação 2.1. Outra forma de obter o grupo de Lie $\mathbb{S}^{1}$ é considerar o subgrupo normal discreto $\mathbb{Z}$ em $\mathbb{R}$ com a operação adição. O quociente é uma variedade

$$
\mathbb{R} / \mathbb{Z} \simeq \mathbb{S}^{1}
$$

com uma operação suave induzida pela adição de $\mathbb{R}$.

Proposição 2.2. Seja G um grupo de Lie de dimensão n, abeliano e conexo. Então

$$
G \simeq \mathbb{T}^{k} \times \mathbb{R}^{n-k}
$$

Então todo grupo de Lie compacto, conexo e abeliano é isomorfo a um toro. Um toro contido em $G$ é maximal se não está propriamente incluído em nenhum outro toro.

Exemplo 2.3. Os complexos unitários $\mathbb{S}^{1}$ formam um toro maximal nos quaternions unitários $\mathbb{S}^{3}$.

Considere uma variedade riemanniana $(M, g)$ e um grupo de Lie $G$ com identidade $e$. Uma aplicação

$$
\mu: G \times M \rightarrow M
$$

é uma $a c ̧ \tilde{a} o$ (à esquerda) se, para todo $x \in M$ e todos $g, h \in G$,

$$
\mu(e, x)=x \quad \text { e } \quad \mu(g, \mu(h, x))=\mu(g \cdot h, x) .
$$

Isso nos dá um homomorfismo

$$
\begin{aligned}
& \rho: G \rightarrow \operatorname{Diff}(M) \\
& g \mapsto \rho(g): M \quad \rightarrow \quad M \\
& x \mapsto \mu(g, x),
\end{aligned}
$$

onde $\operatorname{Diff}(M)$ é o grupo de difeomorfismos de $M$. É também comum neste contexto chamar $M$ de $G$-variedade.

Cada $\xi \in \mathfrak{g}$ induz um campo suave em $M$ dado por

$$
\left(X^{\xi}\right)_{p}:=d \rho_{e}(\xi)=\left.\frac{d}{d t} \mu(\exp (t \xi), p)\right|_{t=0},
$$




\subsection{Ações}

cujo fluxo é $\varphi_{t}(p)=\mu(\exp (t \xi), p)$.

Desta forma, uma ação $\rho$ induz um anti-homomorfismo entre $\mathfrak{g}$ e $\Xi(M)$, uma vez que

$$
\left[X^{\xi}, X^{\eta}\right]=-X^{[\xi, \eta]}
$$

para todos $\xi, \eta \in \mathfrak{g}$.

Uma ação é própria se a aplicação

$$
\begin{aligned}
G \times M & \rightarrow M \times M \\
(g, x) & \mapsto(\mu(g, x), x)
\end{aligned}
$$

for própria.

Exemplo 2.5. Se $G$ é compacto, a ação de $G$ em $M$ é própria.

Dada uma ação de $G$ em $M$, a órbita de um ponto $x \in M$ é

$$
G(x):=\{\mu(g, x): g \in G\} .
$$

A variedade $M$ é a união disjunta das órbitas da ação. Se há apenas uma órbita, a ação é dita transitiva.

Exemplo 2.6. A ação de $S O(n+1)$ em $\mathbb{S}^{n}$ - restrição da ação natural de $G L\left(\mathbb{R}^{n+1}\right)$ em $\mathbb{R}^{n+1}$ - é transitiva.

O subgrupo de isotropia da ação em $x$ (ou estabilizador do ponto $x$ ) é

$$
G_{x}:=\{g \in G: \mu(g, x)=x\} .
$$

Este conjunto é compacto para ações próprias. Se o subgrupo de isotropia é trivial em todo ponto, a ação é dita livre.

Um ação é efetiva se

$$
\bigcap_{x \in M} G_{x}=\{e\}
$$

Observe que toda ação pode ser reduzida a uma ação efetiva tomando-se a ação do quociente de $G$ pelo subgrupo normal dado pela interseção acima (que será, neste caso, não-trivial). Consideraremos então, a partir de agora, apenas ações efetivas.

Temos que $G_{x}$ é um subgrupo fechado de $G$ e o quociente $G / G_{x}$ é uma variedade. Para cada $x \in M$, a aplicação suave definida por $\omega_{x}(g):=\mu(g, x)$ é constante em $G_{x}$ e tem por imagem a órbita de $x$. Assim, $\omega_{x}$ induz uma imersão injetiva

$$
G / G_{x} \rightarrow M
$$




\section{Folheações infinitesimalmente polares}

donde conclui-se que as órbitas de uma ação são subvariedades imersas. Se a ação for própria, vale que as órbitas são mergulhadas.

Uma órbita $G(p)$ é dita principal se existe vizinhança $U$ de $p$ tal que, se $q \in U, G_{p} \subseteq G_{\mu(g, q)}$ para algum $g \in G$. Ou seja, são órbitas que tem, em certo sentido, um grupo de isotropia menor que o de órbitas próximas.

Lembre que o grupo de isometrias de uma variedade riemanniana conexa $M$, Iso $(M)$, é um grupo de Lie (com a topologia da convergência compacta e composição de aplicações como operação). Se $G$ é um subgrupo de $\operatorname{Iso}(M)$, a ação de $G$ em $M$ será chamada de isométrica. Alguns autores chamam ações isométricas de ações riemannianas. Ações isométricas e ações próprias são, essencialmente, o mesmo tema, como fica claro pelos dois itens abaixo.

Exemplo 2.7. Se $G$ é um subgrupo fechado de $\operatorname{Iso}(M)$, então a ação de $G$ em $M$ é própria.

Lema 2.8. Se a ação de $G$ em $M$ é própria, então existe uma métrica em $M$ tal que que $G$ é subgrupo fechado de $\operatorname{Iso}(M)$. (Mais precisamente: $\mu(G, \cdot) \prec \operatorname{Iso}(M)$.)

Observe que se a ação de $G$ é isométrica, para todo $\xi \in \mathfrak{g}$ o campo induzido $X^{\xi}$, vide equação (2.4), é um campo de Killing.

Definição 2.9. Uma ação isométrica de de $G$ em $M$ é dita polar se existe uma subvariedade imersa e completa que encontra todas as órbitas de forma que a interseção seja ortogonal. Tal subvariedade é chamada seção. Se a seção for flat, a ação é chamada de hiperpolar.

Uma seção $i: \Sigma \hookrightarrow M$ é sempre totalmente geodésica e os pontos regulares em $\Sigma$ são abertos e densos em $\Sigma$. A imersão $i$ pode ser tomada de modo a garantir injetividade nos pontos regulares.

Se $p \in M$ for regular pela ação de $G, G(p)$ tem dimensão máxima e existe única seção $\Sigma$ que contém $p$. Assim, é fácil ver que

$$
\Sigma=\exp \left(\nu_{p} G(p)\right)
$$

Exemplo 2.10. Uma ação isométrica que tenha uma hiperfície como órbita é polar. Uma geodésica ortogonal a esta órbita é ortogonal a todas as órbitas e logo uma seção.

Considere $G$ agindo por isometrias em $M$. Um campo de Jacobi em $M$ é $G$-tranversal se for variação infinitesimal de uma família de geodésicas todas perpendiculares às órbitas. 


\subsection{O exemplo clássico de ação polar}

Definição 2.11. Uma ação isométrica de $G$ é variacionalmente completa se todo campo de Jacobi $G$-transversal que é tangente a órbitas em dois pontos distintos é restrição de um campo de Killing induzido pela ação.

Mais precisamente, se $J$ é campo de Jacobi $G$-tranversal sobre $\gamma$ com

$$
J\left(t_{0}\right) \in T_{\gamma\left(t_{0}\right)} G\left(\gamma\left(t_{0}\right)\right) \quad \text { e } \quad J\left(t_{1}\right) \in T_{\gamma\left(t_{1}\right)} G\left(\gamma\left(t_{1}\right)\right),
$$

então

$$
J(t)=X^{\xi}(\gamma(t))
$$

Na seção 3.4 iremos discutir com maior detalhes esta classe de ações. Por ora, vamos apenas listar alguns resultados que relacionam o caso variacionalmente completo com os anteriores.

Proposição 2.12. Ações hiperpolares de grupos compactos são variacionalmente completas.

Proposição 2.13. Ações polares de grupos compactos cujas seções são livres de pontos conjugados são variacionalmente completas.

Uma variedade riemanniana $M$ é um espaço simétrico se a inversão de geodésicas em todo ponto é uma isometria, ou seja, para todo ponto $p$ em $M$ existe uma isometria que fixa $p$ e cuja derivada em $p$ é $-I$. Tal isometria recebe o nome de simetria.

Teorema 2.14. Ações variacionalmente completas em espaços simétricos compactos são hiperpolares.

O teorema 3.24 será outra recíproca da proposição 2.12 .

\subsection{O exemplo clássico de ação polar}

Nesta seção, vamos relembrar o clássico teorema do toro maximal. Os resultados apresentados no resto deste texto, em grande parte, podem ser encarados como generalizações desta situação modelo. Uma métrica em $G$ é dita biinvariante se é invariante por translações à direita e à esquerda; ver detalhes, por exemplo, em Alexandrino e Bettiol [5], capítulo 2. É possível mostrar que todo grupo compacto admite uma tal métrica (que pode ser definida explicitamente).

Primeiro, vamos lembrar a representação isotrópica de uma ação. Se $p \in M$ é ponto fixo de uma ação $\mu$ de $G, \mu^{g}:=\mu(g, \cdot)$ leva $p$ em $p$ para todo 


\section{Folheações infinitesimalmente polares}

$g \in G, \operatorname{logo}\left(d \mu^{g}\right)_{p}$ é uma transformação linear de $T_{p} M$ e temos uma ação (linear) de $G$ em $T_{p} M$ :

$$
\hat{\mu}(g, v):=\left(d \mu^{g}\right)_{p} v .
$$

Em geral, para qualquer $p \in M$, de forma análoga, define-se uma ação linear de $G_{p}$ em $T_{p} M$ chamada representação isotrópica em $p$.

Proposição 2.15. Representações isotrópicas de uma ação polar são polares.

Um grupo de Lie age de maneira natural em si mesmo através da $a c ̧ a ̃ o$ por conjugação:

$$
\begin{aligned}
a: G \times G & \rightarrow G \\
(g, h) & \mapsto g h g^{-1} .
\end{aligned}
$$

É claro que o elemento neutro e é um ponto fixo e a ação por conjugação induz ação de $G$ em $\mathfrak{g}=T_{e} G$

$$
A d(g) X:=\left(d a^{g}\right)_{e} X
$$

neste caso chamada representação adjunta.

Se $G$ é compacto e conexo, existe toro maximal $T \subseteq G$ e dois toros maximais em $G$ estão conjugados. Mais ainda, todo elemento de $G$ está contido em um toro maximal.

Segue que, fixando-se um toro maximal $T$, todo elemento em $G$ está conjugado a um elemento em $T$. Mais ainda, a interseção de $T$ com uma órbita qualquer é de forma ortogonal e $T$ é flat (ver, por exemplo, Alexandrino e Bettiol [5]), donde a ação por conjugação é hiperpolar.

Na situação acima, a exponencial de Lie é uma correspondência biunívoca entre toros maximais de $G$ e subespaços abelianos maximais de $\mathfrak{g}$.

Cada um desses subespaços maximais $\mathfrak{t}$ é uma seção (flat) para a ação adjunta.

Em Bott [9], é demonstrado diretamente que a ação por conjugação e a ação adjunta são variacionalmente completas.

Esta situação foi posteriormente generalizada pelas proposições $2.12 \mathrm{e}$ 2.15 .

É fácil ver que órbitas principais da representação adjunta são isoparamétricas, tal fato também foi posteriormente generalizado na proposição seguinte.

Proposição 2.16. As órbitas principais de ações polares em formas espaciais são isoparamétricas. 


\subsection{O exemplo clássico de ação polar}

A representação adjunta tem uma notável propriedade que não se generaliza: todas as órbitas regulares são principais. Em geral, ações polares podem admitir órbitas exóticas de máxima dimensão.

Considere $\mu$ ação polar de $G$ em $M$ completa e $\Sigma$ uma seção. Seja $N(\Sigma)$ o normalizador de $\Sigma$, isto é,

$$
N(\Sigma):=\{g \in G: \mu(g, \Sigma) \subseteq \Sigma\}
$$

e $Z(\Sigma)$ o centralizador de $\Sigma$, isto é,

$$
Z(\Sigma):=\{g \in G: \mu(g, x)=x, x \in \Sigma\} .
$$

O grupo de Weyl (generalizado) da ação é

$$
W:=N(\Sigma) / Z(\Sigma)
$$

que não depende da seção escolhida e é discreto.

O fato interessante é que a ação propriamente descontinua de $W$ em $\Sigma$ guarda muita informação da ação original. As órbitas desta ação são precisamente as interseções das órbitas de $G$ em $M$ na seção:

$$
G(x) \cap \Sigma=W(x),
$$

para todo $x \in \Sigma$. Neste sentido, uma vez a seção intersecta todas as órbitas, temos uma equivalência entre os quocientes (ver seção 2.4)

$$
\Sigma / W \rightarrow M / G
$$

O estudo de raízes em grupos compactos, conexos e simples permite uma classificação completa dos mesmos. Não vamos abordar este assunto aqui, mas vale destacar o seguinte comportamento da representação adjunta em uma seção, que também é observado, em sentido mais amplo, para certas folheações.

O grupo de Weyl $W \subset G$ é o mesmo para as duas ações acima. Considerando a representação adjunta, é possível obter um número finito de funcionais lineares de $\mathfrak{g}$ chamados raízes de $G$, associados a subespaços de $\mathfrak{g}$ (seus núcleos). Na seção $\mathfrak{t}$, tem-se que a interseção com estes subespaços é exatamente a interseção com as órbitas singulares. Estas interseções são chamadas paredes e definem um número finito de componentes convexas no seu complementar em $\mathfrak{t}$. O fecho de uma destas componentes é uma câmara de Weyl e intersecta cada órbita da representação adjunta precisamente uma vez. Finalmente, vale que $W$ é gerado pelas reflexões nas paredes e uma câmara de Weyl é um domínio fundamental para sua ação em $\mathfrak{t}$.

Uma aplicação destas idéias num exemplo prático é dada na seguinte observação. 


\section{Folheações infinitesimalmente polares}

Observação 2.18. Um teorema de álgebra linear básica garante que toda matriz unitária é conjugada a uma matriz diagonal, isto é, se $m$ é unitária, existe $b$ unitária tal que

$$
b \cdot m \cdot b^{-1}
$$

é diagonal com os autovalores de $m$ como suas entradas.

Observe que as matrizes unitárias $n \times n$ formam um grupo de Lie $U(n)$ e as matrizes diagonais formam toro maximal $T \subseteq U(n)$.

O grupo de Weyl é o grupo simétrico de $n$ elementos e age em $T$ permutando as entradas das matrizes diagonais, o que representa as diferentes formas de se diagonalizar $m$. (Estas permutações podem ser realizadas através de multiplicação por certas matrizes.)

Um elemento é regular se todos os autovalores forem distintos e singular caso contrário. Veja que, do ponto de vista da álgebra linear, uma matriz em uma órbita singular está conjugada a uma mesma matriz diagonal por diferentes mudanças de base.

\subsection{Folheações singulares}

Uma folheação regular é uma decomposição de uma variedade em subvariedades bem-comportadas de mesma dimensão, de modo que localmente se tenha uma situação difeomorfa a decomposição de um espaço euclideano por planos paralelos. Introduziremos o termo singular para permitir decomposições que admitam folhas de diferentes dimensões.

Em analogia à idéia acima, uma folheação riemanniana singular é uma decomposição em subvariedades de modo que localmente se tenha uma situação difeomorfa a um cilindro sobre uma esfera como ficará claro na seção 2.5 .

Definição 2.19. Uma folheação singular $\mathcal{F}$ em uma variedade $(M, g)$ é uma partição de $M$ em subvariedades conexas, imersas e sem auto-interseções chamadas folhas - tal que todo vetor tangente a uma folha pode ser estendido a um campo em $M$ tangente às folhas.

Por cada $p \in M$ passa uma única folha que será notada $L_{p}$. Uma folha é regular se sua dimensão for máxima e singular caso contrário. Um ponto $p$ é regular se $L_{p}$ for regular e singular caso contrário.

A definição de singular pode ser posta mais precisamente: seja $p \in M \mathrm{e}$ $v \in T_{p} L_{p}$, existe $X \in \Xi(M)$ tal que $X_{p}=v$ e $X_{q} \in T_{q} L_{q}$ para todo $q \in M$. O conjunto $\Xi(\mathcal{F})$ dos campos em $\Xi(M)$ que são sempre tangentes às folhas forma um módulo sobre $\mathcal{C}^{\infty}(M)$. A condição acima significa dizer que $\Xi(\mathcal{F})$ é transitivo em cada folha. 


\subsection{Folheações singulares}

Este conceito pode ser encarado como uma generalização de ações de grupos de Lie, vide próximo exemplo.

Exemplo 2.20. Seja $G$ um grupo de Lie agindo em $M$. Sabemos que as órbitas são subvariedades conexas, imersas e sem auto-interseção. Agora seja $p \in M$ e considere $Y \in T_{p} L_{p}=T_{p} G(p)$. Podemos olhar para $Y$ como vetor tangente $\gamma^{\prime}(0)$ em $p$ de uma curva $\gamma$ em $G(p)=L_{p}$ com $\gamma(0)=p$. Mas uma curva em $G(p)$ se escreve

$$
\mu(\alpha(t), p),
$$

onde $\alpha$ é uma curva em $G \operatorname{com} \alpha(0)=e$. Logo $\alpha^{\prime}(0) \in T_{e} G$ induz um campo $X^{\alpha^{\prime}(0)}$ pela equação $(2.4)$ que em todo ponto é tangente às órbitas e, por construção,

$$
\left(X^{\alpha^{\prime}(0)}\right)_{p}=Y \text {. }
$$

Uma folheação singular pode ser também definida como partição de $M$ por subvariedades integrais maximais de uma distribuição que verifique o teorema de Frobenius generalizado (com singularidades). Esta definição é equivalente à dada acima; uma demonstração deste fato pode ser encontrada em Correa da Silva [18].

Observação 2.21. A definição de folheações (regulares), normalmente feita através de cartas (ver, por exemplo, Molino [27] ou apêndice de Alexandrino e Bettiol [5]), também pode ser dada seguindo esta abordagem, com a ressalva de que todas as folhas devem ter a mesma dimensão. O roteiro para obter uma carta folheada local é o seguinte. Seja $\mathcal{F}$ uma folheação de dimensão $k$ em $M^{n}$. Tome $p \in M$ e fixe uma base de $T_{p} L_{p}$. A definição fornece campos $X_{1}, \cdots, X_{k}$ que coincidem com a base escolhida em $p$ e logo são linearmente independentes num aberto $U \ni p$. Seja $P_{p}$ componente conexa de $\mathcal{F}$ restrita a $U$ que contém $p$. Seja $S_{p}$ uma subvariedade de $M$ transversal a $P_{p}$ que contém em $p$ e está contida em $U$. Considere $\varphi_{t}^{i}$ o fluxo de $X_{i}$ e bola $B_{\varepsilon}(0) \subseteq \mathbb{R}^{k} \mathrm{e}$ defina

$$
\begin{aligned}
F: B_{\varepsilon}(0) \times S_{p} & \rightarrow M \\
\left(\left(t_{1}, \cdots, t_{k}\right), y\right) & \mapsto \varphi_{t_{1}}^{1} \circ \cdots \circ \varphi_{t_{k}}^{k}(y) .
\end{aligned}
$$

Diminuindo $U$ se necessário, $F$ é um difeomorfismo que leva, para todo $y \in$ $S_{p}, B_{\varepsilon}(0) \times\{y\}$ na componente conexa de $\mathcal{F}$ restrita a $U$ que contém $F(0, y)$.

A partir de aplicações locais como $F$ é fácil obter um atlas folheado de $M$ sobre $\mathbb{R}^{n}$.

Uma 1-forma não-singular $\omega$ em uma variedade conexa $M$ define um campo de hiperplanos por

$$
D_{x}:=\operatorname{ker} \omega_{x} .
$$




\section{Folheações infinitesimalmente polares}

Se $\omega \wedge d \omega=0$ (em particular, se $\omega$ for fechada) esta distribuição verifica a condição de integrabilidade do teorema de Frobenius e define uma folheação regular em $M$. Podemos generalizar este cenário para folheações singulares.

Exemplo 2.22. Uma 1-forma $\omega$ fechada qualquer define uma folheação regular em $M \backslash A$, onde

$$
A:=\left\{x \in M: \omega_{x} \equiv 0\right\}
$$

é o conjunto dos pontos onde $\omega$ se anula (singularidades).

Tomando cada $p \in A$ como uma folha (de dimensão 0 ), temos que os campos tangentes às folhas em $M \backslash A$ quando estendidos para $M$ cumprem trivialmente a condição de transitividade nas folhas singulares. Logo $\omega$ define uma folheação singular de codimensão 1.

Exemplo 2.23. Um caso particular do exemplo acima é quando

$$
\omega=d f
$$

para uma função suave $f: M \rightarrow \mathbb{R}$. Neste caso, $A$ é exatamente o conjunto dos pontos críticos de $f$ e as folhas são conjuntos de nível conexos de $f$.

Se $f$ for uma submersão, temos uma folheação regular. Vale observar que não se pode definir folheações regulares desta forma em variedades compactas.

Definição 2.24. Dizemos que a métrica $g$ é adaptada a uma folheação $\mathcal{F}$ se toda geodésica em $(M, g)$ que encontra uma folha ortogonalmente permanece ortogonal a todas as folhas que encontra. Diz-se que $\mathcal{F}$ é uma partição transnormal neste caso.

Transnormalidade implica que as folhas sejam localmente equidistantes, isto é se $L$ e $L^{\prime}$ são folhas e $p \in L$, existe vizinhança $U \subseteq L$ de $p$ em tal que

$$
\operatorname{dist}\left(q, L^{\prime}\right)=\operatorname{dist}\left(p, L^{\prime}\right),
$$

para todo $q \in U$. Se as folhas de $\mathcal{F}$ são propriamente mergulhadas, vale que são (globalmente) equidistantes.

Partições transnormais são também chamadas de riemannianas.

Definição 2.25. Uma folheação singular transnormal será chamada folheação riemanniana singular. As geodésicas ortogonais às folhas serão ditas horizontais.

Exemplo 2.26. Se a ação do exemplo 2.20 for isométrica, a folheação singular será riemanniana. 


\subsection{Quocientes e orbifolds}

Exemplo 2.27. Se $\mathcal{F}$ é uma folheação riemanniana regular em uma variedade completa e compacta $M$. A partição dada pelos fechos das folhas de $\mathcal{F}$ é uma folheação riemanniana singular.

Observação 2.28. O exemplo 2.27 é um resultado de Molino, que conjecturou também ser verdadeira a conclusão no caso em que $\mathcal{F}$ é riemanniana singular. Este problema, nesta generalidade, permanece em aberto como a conjectura de Molino.

A união da folhas de dimensão $r, \Sigma^{r}$, será chamada stratum das folhas de dimensão $r$ e é uma subvariedade mergulhada em $M$. O stratum das folhas de menor dimensão de $\mathcal{F}$ será chamado minimal e é uma subvariedade fechada. O stratum da folhas regulares será chamado regular e denotado $M_{0}$.

Se $\mathcal{F}$ é riemanniana singular em $(M, g)$, então $\mathcal{F}$ restrita ao $\Sigma^{r}$ é uma folheação riemanniana (regular) na métrica induzida por $g$ no stratum.

\subsection{Quocientes e orbifolds}

Nesta seção, vamos introduzir bons orbifolds como espaços que surgem naturalmente como quocientes de ações. Para definirmos uma classe mais geral de espaços, orbifolds não necessariamente bons, vamos relembrar folheações regulares e introduzir seus quocientes. Citamos como referências o apêndice G em Bridson e Haefliger [11], apêndice D de Salem em Molino [27], Borzellino e Brunsden [8] e Thurston [38]. No capítulo 2 em Weilandt [41], há um resumo recente sobre orbifolds.

Como já observado, as órbitas da ação de um grupo $G$ particionam $M$ em subvariedade disjuntas. Podemos introduzir naturalmente uma relação de equivalência em $M$ :

$$
x \sim y \quad \text { se } \quad G(x)=G(y),
$$

e o espaço de órbitas é o espaço das classes de equivalência de $\sim$ denotado pelo quociente $M / G$.

Exemplo 2.29. Se $G$ age de forma própria e livre em $M$, então $M / G$ é uma variedade e $\pi: M \rightarrow M / G$ é suave.

Definição 2.30. Um bom orbifold riemanniano é o quociente de uma variedade riemanniana por um grupo discreto de isometrias que age de forma própria.

Exemplo 2.31 (cone). Seja $D$ disco de raio 1 no plano. Considere $\alpha$ a rotação de $\pi / 2$ (45 graus) ao redor da origem (o exemplo é idêntico para os 


\section{Folheações infinitesimalmente polares}

sentidos anti-horário ou horário). É fácil ver que $\alpha$ é uma isometria de $D$ e $\alpha^{2}=\alpha \circ \alpha$ é uma rotação de $\pi ; \alpha^{3}$ é uma rotação de $-\pi / 2$ e $\alpha^{4}=$ id é uma rotação de 0 . Então $\alpha$ gera um grupo de isometrias $\Gamma$ de $D$ com quatro elementos (isomorfo a $\mathbb{Z}_{4}$ ).

A figura que obtemos com $D / \Gamma$ é um cone. A ponta do cone é a imagem da origem no quociente. É interessante notar que para um cone ser uma variedade, devemos omitir a singularidade, isto é, a ponta. Este detalhe impede que se tenha uma variedade completa. O cone com sua ponta é um bom orbifold.

Note que tomando o grupo (isomorfo a $\mathbb{Z}_{p}$ ) gerado por uma rotação de $2 \pi / p$, temos que $D / \mathbb{Z}_{p}$ é igualmente um cone.

O exemplo acima indica também que localmente o quociente pode ter uma estrutura mais bem comportada. A menos de um ponto, todo ponto em $D / \Gamma$ admite uma vizinhança que é localmente uma variedade.

Um ponto será chamado ponto orbifold se possui vizinhança que é um bom orbifold. Analogamente, um ponto manifold possui uma vizinhança que é uma variedade.

Observação 2.32. Se $M / G$ é um orbifold bom, então o estabilizador $G_{x}$ é finito para todo $x \in M$.

Interessante que diversos espaços que surgem naturalmente em geometria não são variedades, mas estão na classe dos orbifolds: variedade-com-cantos, variedade-com-bordo, cone, hemisfério e outros exemplos serão exibidos nesta seção.

Exemplo 2.33 (bola de futebol americano). Seja $M=\mathbb{S}^{2} \subset \mathbb{R}^{3}=\{(x, y, z)\}$ e considere $G$ o grupo de rotações (que são isometrias) em torno do eixo $z$ gerado por uma rotação de ângulo $\frac{2 \pi}{p}$ para $p>1$ inteiro.

Agora $A F(p):=M / G$ é um bom orbifold. As únicas singularidades estão nos pólos $p_{1}=(0,0,1)$ e $p_{2}=(0,0,-1)$ que são fixados por $G$. Este pontos são, de fato, os únicos que tem estabilizador não-trivial:

$$
G_{p_{1}}=G_{p_{1}}=G \simeq \mathbb{Z}_{p}
$$

A figura obtida se assemelha a uma bola de futebol americano.

Exemplo 2.34 (semi-reta). A semi-reta $M=[0, \infty)$ é o quociente da reta $\mathbb{R}$ pelo grupo formado por $x \mapsto x$ e $x \mapsto-x$. Logo é um bom orbifold. Por outro lado, é também uma variedade com bordo $\partial M=\{0\}$.

Exemplo 2.35 (semi-espaço). O semi-espaço $M^{n}:=\left\{\left(x_{0}, \cdots, x_{n-1}\right) \in \mathbb{R}^{n}\right.$ : $\left.x_{0}>0\right\}$ é idêntico ao caso acima, com $\partial M=\left\{x_{0}=0\right\} \simeq \mathbb{R}$. 


\subsection{Quocientes e orbifolds}

Exemplo 2.36 (hemisfério). Seja $M=\mathbb{S}^{2} \subset \mathbb{R}^{3}=\{(x, y, z)\}$ e considere $G \simeq \mathbb{Z}_{2}$ gerado pela reflexão em torno do plano $\{z=0\}$. Quando restrito a esfera, $G$ equivale ao grupo de isometrias gerado por reflexões em torno de um grande círculo.

As órbitas singulares da ação são os pontos de $\left\{x^{2}+y^{2}=1\right\}$. O quociente é topologicamente um disco fechado. A métrica induzida no quociente pela métrica canônica na esfera faz de $M / G$ um espaço com curvatura constante 1.

Exemplo 2.37 (variedade-com-cantos). Este é uma variação exemplo anterior. Tome $\mathbb{S}^{n} \hookrightarrow \mathbb{R}^{n+1}=\left\{\left(x_{1}, \cdots, x_{n}\right)\right\}$ e $\Gamma$ o grupo gerado por todas as reflexões em todos os $n$-planos da forma $\left\{x_{i}=0\right\}$. Este quociente é um orbifold bom de dimensão $n$ com $n$ singularidades do tipo ponto (dimensão 0 ), bem como singularidades de dimensão $k$ para $k<n$.

Observe que, para $n=1$, temos topologicamente um intervalo fechado, isto é, uma variedade-com-bordo. Este quociente, porém, tem curvatura constante 1 herdada da esfera.

Para $n=2$, o quociente é topologicamente um triângulo, isto é, tem singularidades de dimensão 0 (vértices) e 1 (arestas).

Em geral, $n$-variedades-com-cantos são espaços localmente homemorfos a $[0, \infty)^{k} \times \mathbb{R}^{n-k}$ para diferentes $k$. Maiores detalhes podem ser encontrados, por exemplo, em Joyce [22].

O espaço de órbitas pode ter uma estrutura global muito complicada. É difícil termos uma estrutura de variedade. Vamos agora partir para obter uma definição mais ampla de orbifold (não necessáriamente bom).

Uma folheação (regular) é localmente entendida por cartas onde as folhas são dadas fazendo as últimas $k$ coordenadas constantes. Globalmente, isto significa que tem-se um atlas sobre a variedade satisfazendo certas condições de compatibilidade. Esta abordagem a seguir é equivalente à dada por cartas locais.

Uma folheação $\mathcal{F}$ (regular) de codimensão $k$ em $M$ é uma cobertura de $M$ por abertos $\left\{U_{i}\right\}_{i \in I}$ e submersões

$$
f_{i}: U_{i} \rightarrow \sigma_{i}
$$

onde $\sigma_{i}$ é uma subvariedade de dimensão $k$ e, para toda interseção não-vazia $U_{i} \cap U_{j}$, temos trocas

$$
\omega_{i j}: f_{i}\left(U_{i} \cap U_{j}\right) \subseteq \sigma_{i} \rightarrow f_{j}\left(U_{i} \cap U_{j}\right) \subseteq \sigma_{j},
$$

tais que

$$
f_{j}=\omega_{i j} \circ f_{i}
$$




\section{Folheações infinitesimalmente polares}

em $U_{i} \cap U_{j}$.

As folhas de $\mathcal{F}$ são as componentes conexas de $M$ na topologia cuja base de abertos é dada pelos abertos das fibras das submersões $f_{i}$. As folhas são subvariedades imersas de codimensão $k$.

Se os $\omega_{i j}$ são isometrias, a folheação é dita riemanniana. E os $\omega_{i j}$ agindo em

$$
\sigma:=\bigcup_{i \in I} \sigma_{i}
$$

geram um pseudogrupo $\Phi$ de isometrias de $\sigma$ : o pseudogrupo de holonomia de $\mathcal{F}$.

Vamos relembrar agora a definição de pseudogrupo. Um pseudogrupo é um objeto algébrico que generaliza a noção de grupo de difeomorfismos (ou homeomorfismos) de uma variedade (ou espaço topológico). Estamos interessados somente no caso riemanniano, isto é, coleções de isometrias locais.

Definição 2.38. Um pseudogrupo riemanniano é uma coleção $W$ de isometrias locais de uma variedade $\Sigma$ (possivelmente desconexa) da forma $\omega: U \rightarrow$ $V$, onde $U$ e $V$ são abertos de $\Sigma$ tais que

(i) se $\omega \in W$, então $\omega^{-1} \in W$;

(ii) se $\omega, \psi \in W$, então $\psi \circ \omega \in W$ onde a composição fizer sentido;

(iii) se $\omega \in W$, então sua restrição a qualquer aberto em seu domínio também está em $W$;

(iv) se $\omega$ é uma isometria entre abertos de $\Sigma$ tal que todas as suas restrições locais coincidem com um elemento de $W$, então $\omega \in W$;

(v) a identidade de $\Sigma$ está em $W$.

Se $A$ é uma coleção de isometrias de uma variedade $\Sigma$ contendo a identidade, então tomando todas as inversas, uniões, restrições a abertos e composições dos elementos de $A$ obtemos o psedogrupo gerado por $A$.

Definição 2.39. Sejam $\Sigma$ e $\sigma$ duas variedade com pseudogrupos riemannianos $W$ e $\Phi$, respectivamente. Dizemos que $(\Sigma, W)$ e $(\sigma, \Phi)$ são equivalentes se existe uma coleção $\Psi$ de isometrias de abertos de $\Sigma$ para abertos de $\sigma$ tal que

(i) $\Psi$ é maximal;

(ii) os domínios dos elementos de $\Psi$ cobrem $\Sigma$;

(iii) os contra-domínios dos elementos de $\Psi$ cobrem $\sigma$; 


\subsection{Quocientes e orbifolds}

(iv) se $\psi_{\alpha}, \psi_{\beta} \in \Psi, \omega \in W, \varphi \in \Phi$, então

$$
\begin{aligned}
\varphi \circ \psi_{\alpha} \circ \omega & \in \Psi ; \\
\psi_{\beta} \circ \omega \circ\left(\psi_{\alpha}\right)^{-1} & \in \Phi ; \\
\left(\psi_{\alpha}\right)^{-1} \circ \varphi \circ \psi_{\beta} & \in W .
\end{aligned}
$$

O espaço de órbitas de um pseudogrupo $W$ em uma variedade $\Sigma$, denotado pelo quociente $\Sigma / W$ é o conjuntos das classes de equivalência da relação em $\Sigma$ :

$$
x \sim y \quad \text { se } \omega(x)=y,
$$

para alguma isometria $\omega \in W$ definida em uma vizinhança de $x$. Se $(\Sigma, W)$ e $(\sigma, \Phi)$ são equivalentes, então $\Sigma / W$ e $\sigma / \Phi$ são isomorfos.

Definição 2.40. Um orbifold riemanniano $n$-dimensional é uma classe de equivalência de pseudogrupos de isometrias $W$ de uma variedade $\Sigma^{n}$ tal que

(i) o espaço de órbitas $[\Sigma / W]$ é Hausdorff;

(ii) para todo $y \in \Sigma$, existe vizinhança de $y$ onde a restrição de $W$ é gerada por finitas isometrias.

Observação 2.41. A noção de orbifold apareceu pela primeira vez em Satake [33]. O nome orbifold e a abordagem usual são encontrados originalmente em Thurston [38]. O roteiro que adotamos nesta seção vem do apêndice de Eliane Salem em Molino [27], que segue a abordagem desenvolvida por André Haefliger para pseudogrupos de isometrias.

Vamos agora listar dois exemplos de orbifolds que não são bons, isto é, estes dois exemplos não podem ser obtidos da forma $\mathbb{S}^{2} / \Gamma$.

Exemplo 2.42 (gota). A esfera $\mathbb{S}^{2}$ com uma singularidade. O ponto singular é um ponto orbifold: tem uma vizinhança $\simeq \mathbb{R}^{2} / \mathbb{Z}_{p}$, onde $\mathbb{Z}_{p}$ é grupo de $p$ rotação ao redor da origem ( $\simeq$ ponto singular).

A gota também pode ser obtida colando-se um cone com um hemisfério ao longo de um círculo.

Exemplo 2.43 (bola de futebol americano ruim). A idéia acima pode ser generalizada para múltiplas singularidades. Tomando-se duas singularidades a partir de pontos antipodais da esfera com vizinhanças do tipo $\mathbb{R}^{2} / \mathbb{Z}_{p}$ e $\mathbb{R}^{2} / \mathbb{Z}_{q}$, para $p \neq q$, temos uma bola oval ruim. Este orbifold é comumente chamado de $A F(p, q)$.

Pontos orbifolds como as singularidades dos dois exemplos acima são chamados singularidades cônicas, por analogia ao exemplo 2.31 . 


\section{Folheações infinitesimalmente polares}

Proposição 2.44. Seja $\mathcal{F}$ uma folheação riemanniana regular com folhas fechadas e mergulhadas (por exemplo, folhas compactas) em uma variedade $M$ completa. Então o espaço de folhas $M / \mathcal{F}$ é um orbifold riemanniano e

$$
M / \mathcal{F} \simeq \sigma / \Phi
$$

onde $\Phi$ é o pseudogrupo de holonomia de $\mathcal{F}$ agindo em $\sigma$ definidos acima nesta seção.

Observação 2.45. Vale a volta da proposição 2.44, isto é, dado um orbifold riemanniano $A$, existe uma folheação riemanniana com folhas compactas em uma variedade completa $M$ tal que $A \simeq M / \mathcal{F}$. Para mais detalhes nesta direção, ver Moerdijk e Mrcun [26].

\subsection{Vizinhança tubular}

O estudo de propriedade locais de folheações riemannianas singulares é facilitado por um resultado clássico que fornece, para qualquer ponto, uma vizinhança com características muito convenientes. Esta seção tem por objetivo apresentar (ou relembrar) este resultado. A vizinhança $O$ que será construída abaixo é chamada de vizinhança tubular distinguida - também chamada, por alguns autores, de vizinhança tubular trivializadora ou de Molino. Para maiores detalhes (e algumas demonstrações) sobre os resultados aqui apresentados, sugerimos Molino [27], Alexandrino e Toeben [6] ou Piñeros [32].

Seja $\mathcal{F}$ uma folheação riemanniana singular em $M$. Seja $x \in M$ e considere $P$ subconjunto aberto, conexo e relativamente compacto contendo $x$ na folha $L_{x}$ de $x$. (Um conjunto com estas propriedades será chamado de placa contendo $x$.) No fibrado normal de $L_{x}$, para $\rho>0$, seja $N^{\rho}(P)$ fibrado de bolas abertas sobre $P$ dado pelos vetores normais à folha $L_{x}$ nos pontos de $P$.

Considere a vizinhança tubular de $P$ em $M$ dada por

$$
O:=\exp \left(N^{\rho}(P)\right)
$$

Para $\rho$ pequeno, a exponencial normal exp acima é um difeomorfismo e definimos uma transformação tipo homotetia $h_{\lambda}$ na vizinhança tubular conjugando a exponencial com homotetias do fibrado normal:

$$
h_{\lambda}(\exp (v))=\exp (\lambda v)
$$

para todo $v \in N^{\rho}(P)$. 


\subsection{Vizinhança tubular}

Proposição 2.47. A aplicação $h_{\lambda}$ preserva $\mathcal{F}$ em $O$.

O resultado acima, conhecido como lema de transformação homotética de Molino (ver Molino [27], capítulo 6), é a peça fundamental para que possa utilizar mudanças de métrica no sentido de obter resultados sobre folheações riemannianas.

Existe uma projeção-pé $\Pi: O \rightarrow P$ bem-definida: projeção ao longo de geodésicas horizontais. E para $q \in P$, chamamos

$$
S_{q}:=\Pi^{-1}(q)
$$

de um slice. Observe que, para $p \in O, \Pi^{-1}(\Pi(p))$ é slice que contém $p$.

Lema 2.48. A projeção П é um submersão sobrejetora e induz uma folheação em $O$ cujas folhas são os slices.

Se $q \in O$, a placa $P_{q}$ em $O$ que contém $q$ está contida no tubo de raio $\delta<\rho$

$$
\operatorname{Tub}_{\delta}(P):=\{q \in O: \operatorname{dist}(q, P)=\delta\}
$$

isto é

$$
\operatorname{dist}\left(q^{\prime}, P\right)=\operatorname{dist}(q, P), \quad q^{\prime} \in P_{q} .
$$

Observação 2.49. Notamos que, dada uma vizinhança tubular como acima de um ponto singular de uma folheação singular qualquer, a propriedade ser riemanniana é equivalente ao fato das placas estarem contidas nos tubos.

Lema 2.50. A projeção $\left.\Pi\right|_{P_{q}}$ é um submersão sobrejetora e para todo ponto de $P$, o slice que o contém encontra todas as placas em $O$.

Uma geodésica perpendicular a um stratum $\Sigma^{r}$ é também perpendicular às folhas de dimensão $r$. Como geodésicas realizam localmente distâncias, folheações riemannianas têm a seguinte propriedade.

Lema 2.51. As folhas de $\mathcal{F}$ estão contidas (localmente) em tubos (como os definidos anteriormente nesta seção) centrados em $\Sigma^{r}$.

É possível definir uma transformação homotética como (2.46) com respeito ao stratum que também preserva $\mathcal{F}$. A partir daí, com uma consideração dimensional, é possível concluir o seguinte.

Lema 2.52. Todo stratum singular $\Sigma^{r}$ verifica $\operatorname{codim}\left(\Sigma^{r}\right) \geq 2$. E o stratum regular $M_{0}$ é aberto, conexo e denso em $M$.

Observação 2.53. O stratum maximal é também chamado de stratum aberto, em oposição ao stratum minimal, que é fechado. 


\section{Folheações infinitesimalmente polares}

Em Alexandrino e Toeben [6], são apresentados melhoramentos para alguns resultados de Molino, conforme apresentamos abaixo.

Proposição 2.54. Dado $x \in M$, existe vizinhança tubular $O$ de $P$ (como acima) e métricas $\tilde{g}$ e $\hat{g}$ em $O$ tais que

(i) a folheação restrita à vizinhança tubular $\mathcal{F} \cap O$ é folheação riemanniana singular para as métricas $\tilde{g}$ e $\hat{g}$;

(ii) a folheação restrita a um slice $\mathcal{F} \cap S_{q}$ é folheação riemanniana singular nas métricas $\tilde{g}$ e $\hat{g}$, para todo $q \in P$;

(iii) as geodésicas ortogonais à placa $P$ na métrica $g$ continuam ortogonais à $P$ com respeito à métrica $\tilde{g}$ ou à métrica $\hat{g}$;

(iv) para todo $q \in O$, o espaço normal da folha $L_{q}$ (com respeito à métrica $\tilde{g}$ ou à métrica $\hat{g}$ ) é tangente ao slice que contém $q S_{\Pi(q)}$;

(v) a distância entre placas em $O$ é a mesma para a métrica $\tilde{g}$ e para a métrica original $g$;

(vi) para todo $q \in P$,

$$
\exp _{q}:\left(T_{q} S_{q}, \tilde{g}_{q}\right) \rightarrow\left(S_{q}, \hat{g}\right)
$$

é uma isometria.

\subsection{Linearização}

Se $\mathcal{F}$ é uma folheação riemanniana singular em $(M, g)$, é possível, a partir da proposição 2.54, obter uma linearização de $\mathcal{F}$ no sentido em que temos, para todo $x \in M$ singular, uma folheação riemanniana singular $\hat{\mathcal{F}}=\hat{\mathcal{F}}(x)$ em uma vizinhança da origem em um espaço euclideano, também chamada folheação infinitesimal de $\mathcal{F}$ no ponto $x$.

Fixe $x \in M, O$ sua vizinhança tubular distinguida e $S_{x}$ slice com $m:=$ $\operatorname{dim} S_{x}$. Considere a folheação riemanniana singular $\left.\mathcal{F}\right|_{S_{x}}$ na métrica $\hat{g}$ da proposição 2.54. Podemos levantar $\mathcal{F}$ por uma isometria (aplicação exponencial):

$$
\hat{\mathcal{F}}:=A^{-1} \exp ^{-1}(\mathcal{F})
$$

é uma folheação riemanniana singular em $\mathbb{R}^{m}$. A matriz $A$ aqui representa a escolha de uma base no espaço vetorial $\left(T_{x} S_{x}, \tilde{g}_{x}\right)$, desta forma, podemos considerar a folheação $\hat{\mathcal{F}}$ no espaço euclideano $\left(\mathbb{R}^{m}\right.$, can). Observe que se $x$ era ponto singular, temos que $0 \in \mathbb{R}^{m}$ é uma folha de $\hat{\mathcal{F}}(x)$. Notamos que 


\subsection{Uma mudança de métrica local}

$A$ pode ser escolhida de modo que $A^{-1} \exp ^{-1}\left(S_{x}\right)$ seja uma bola centrada na origem.

Para mais detalhes sobre esta construção, ver Alexandrino e Toeben [6], de onde citamos os dois resultados abaixo.

Lema 2.55. A folheação induzida na esfera $\mathbb{S}^{m-1}$ (restrição de $\hat{\mathcal{F}}$ acima) é ainda uma folheação riemanniana singular.

Proposição 2.56. Seja $\mathcal{F}$ uma folheação riemanniana singular em $M$ e $\gamma$ uma geodésica ortogonal a uma folha regular de $\mathcal{F}$. Então os pontos singulares de $\mathcal{F}$ sobre $\gamma$ são isolados.

Vale dizer que a informação relevante sobre a folheação está contida no slice, no sentido em que podemos linearizar a vizinhança tubular $O$ com um difeomorfismo

$$
\phi: O \rightarrow \phi(O) \subseteq \mathbb{R}^{m} \times \mathbb{R}^{n-m},
$$

tal que no primeiro fator temos a linearização do slice acima e o segundo é trivial (vide primeiro caso da observação 2.73).

\subsection{Uma mudança de métrica local}

Fixe $p \in M$. Seja $h_{\lambda}$ a transformação definida pela equação (2.46) para uma placa $P$ que contém $p$. Vamos chamar também de $h_{\lambda}$ a restrição da homotetia ao slice $S=S_{p}$. Para $\lambda<1$, temos $S^{\lambda}:=h_{\lambda}(S) \subseteq S$ e podemos definir

$$
g_{\lambda}:=\frac{1}{\lambda^{2}}\left(h_{\lambda}\right)^{*} \tilde{g}
$$

Lema 2.58. Vale que $\mathcal{F}$ restrita a $\left(S^{\lambda}, g_{\lambda}\right)$ é uma folheação riemanniana singular.

A métrica $g_{\lambda}$ foi estudada por Alexandrino e Toeben [6]. Da demonstração da proposição 2.3 no artigo citado, vemos que

$$
g_{\lambda}\left(\left(d \exp _{p}\right)_{v} X,\left(d \exp _{p}\right)_{v} Y\right)=\tilde{g}\left(\left(d \exp _{p}\right)_{\lambda v} X,\left(d \exp _{p}\right)_{\lambda v} Y\right) .
$$

Assim, podemos falar em $g_{0}$ e fica claro que

$$
g_{0}=\left(\exp _{p}^{-1}\right)^{*} \tilde{g}_{p}
$$

é métrica flat (e restrição ao slice de $\hat{g}$, vide proposição 2.54 ). 


\section{Folheações infinitesimalmente polares}

Seja $m=\operatorname{dim} S$ e $\left\{e_{1}, \cdots, e_{m}\right\}$ a base canônica usual de $\mathbb{R}^{m} \simeq T_{\lambda v} T_{p} S$. Para $1 \leqslant i, j \leqslant m$, definimos aplicação suave $F_{i j}$ por

$$
\left(g_{\lambda}\right)_{i j}(v)=F_{i j}(\lambda, v):=\tilde{g}\left(\left(d \exp _{p}\right)_{\lambda v} e_{i},\left(d \exp _{p}\right)_{\lambda v} e_{j}\right),
$$

então, pelo teorema do valor médio,

$$
\begin{aligned}
\left|F_{i j}(\lambda, v)-F_{i j}(0, v)\right| & \leqslant|| D F_{i j}|| \cdot|\lambda|, \\
\left|\frac{\partial F_{i j}}{\partial v_{i}}(\lambda, v)-\frac{\partial F_{i j}}{\partial v_{i}}(\lambda, v)\right| & \leqslant|| D \frac{\partial F_{i j}}{\partial v_{i}} \| \cdot|\lambda|,
\end{aligned}
$$

Quando restringimos $(\lambda, v)$ ao compacto $\bar{B}_{\delta}(0) \times[0, \varepsilon]$, cada um dos primeiros termos nos lados direitos das equações acima é limitado e temos o seguinte resultado.

Lema 2.60. Quando $\lambda \rightarrow 0, g_{\lambda}$ converge suavemente para a métrica flat

$$
g_{0}=\lim g_{\lambda}=\left(\exp _{p}^{-1}\right)^{*} \tilde{g}_{p}
$$

É possível, de fato, especificar mais o resultado acima. Dada uma folheação $\mathcal{F}$ qualquer em $M$ sempre temos, para todo $x \in M$ uma decomposição trivial

$$
T_{x} M=T_{x} L \oplus T_{x} M / T_{x} L,
$$

onde $L=L_{x}$, mas uma métrica $g$ qualquer em $M$ nos dá a noção de ortogonalidade em cada espaço tangente. Logo podemos decompor

$$
T_{x} M=T_{x} L \oplus T_{x} L^{\perp} .
$$

Considere então para cada métrica $g_{\lambda}$ a decomposição ortogonal correspondente

$$
T_{x} S=\left(T_{x} \hat{L}\right)_{\lambda} \oplus\left(T_{x} \hat{L}\right)_{\lambda}^{\perp} .
$$

É claro que $\left(T_{x} \hat{L}\right)_{\lambda}=T_{x} \hat{L}$ não depende de $\lambda$ (não de depende da métrica). O fato relevante é que a convergência do lema acima respeita esta decomposição em vizinhanças de pontos regulares, isto é,

$$
\left(T_{x} \hat{L}\right)_{\lambda}^{\perp} \rightarrow\left(T_{x} \hat{L}\right)_{0}^{\perp} .
$$

Lembre que a propriedade ser riemanniana para uma folheação depende da métrica ambiente e a geometria transversal da folheação também. Ao mudar a métrica, muda-se o espaço normal e possivelmente a geometria transversal. Assim, como é fundamental a mudança ser para uma métrica compatível, 


\subsection{Uma mudança de métrica local}

isto é, que a folheação seja riemanniana na nova métrica, é necessário manter o controle sobre a geometria tranversal.

Considere

$$
\begin{aligned}
g_{\lambda}^{\top} & :=\left.g_{\lambda}\right|_{T \mathcal{F}} \\
g_{\lambda}^{\perp} & :=\left.g_{\lambda}\right|_{(T \mathcal{F})_{\lambda}^{\perp}},
\end{aligned}
$$

onde as restrições devem ser encaradas levando-se em consideração a decomposição (única) dos vetores tangentes nos dois subespaços ortogonais.

Proposição 2.62. Dados campos linearmente independentes

$$
X_{1}, \cdots, X_{n}
$$

em $S$ tais que, para todo $z$, se $l(z):=\operatorname{dim} L_{z}$,

$$
\left(X_{1}\right)_{z}, \cdots,\left(X_{l(z)}\right)_{z} \in T_{z} L_{z}
$$

Vale que

$$
\begin{aligned}
& g_{\lambda}^{\top} \rightarrow g_{0}^{\top} \\
& g_{\lambda}^{\perp} \rightarrow g_{0}^{\perp} .
\end{aligned}
$$

A idéia da demonstração é obter um referencial ortogonal em relação à métrica $g_{\lambda}$, dependendo suavemente de $\lambda$, que converge a um referencial ortogonal em relação à $g_{0}$. Para isso vamos utilizar o lema 2.60 e o processo Gram-Schmidt.

Observação 2.64. O caso de nosso maior interesse é uma vizinhança de um ponto regular, onde vale a conclusão da proposição. Se $p$ for regular, considere vizinhança $V$ onde $\mathcal{F} \cap S$ é regular (em particular $l(z)=l$ é constante em $V$ ). Tome $X_{1}, \cdots, X_{l} \in \Xi(\mathcal{F})$. Considere campos $X_{l+1}, \cdots, X_{m}$ de forma que, em $p$, se complete uma base de $T_{p} S$. Os campos $X_{1}, \cdots, X_{m}$ permanecem linearmente independentes numa vizinhança $V$ de $p$ e temos o referencial que obedece (2.63) como desejado.

Demonstração da proposição 2.62. Considere os campos dados pelo processo Gram-Schmidt:

$$
\begin{aligned}
& u_{1}^{\lambda}=X_{1} \\
& u_{i}^{\lambda}=X_{i}-\sum_{j=1}^{i-1} \frac{g_{\lambda}\left(X_{i}, u_{j}^{\lambda}\right)}{g_{\lambda}\left(u_{j}^{\lambda}, u_{j}^{\lambda}\right)} u_{j}^{\lambda},
\end{aligned}
$$




\section{Folheações infinitesimalmente polares}

e observe que, para todo $z \in S^{\lambda}$ e todo $\lambda$,

$$
u_{1}^{\lambda}, \cdots, u_{l(z)}^{\lambda}
$$

é base ortogonal do espaço tangente à folha e

$$
g_{\lambda}\left(u_{i}^{\lambda}, u_{j}^{\lambda}\right)=0, \quad i \neq j
$$

$\mathrm{e}$

$$
u_{1}^{\lambda}, \cdots, u_{m}^{\lambda}
$$

é base ortogonal de $T_{z} S$.

Como a convergência de $g_{\lambda}$ é suave, bem como o processo Gram-Schmidt, para todo $z \in S$, temos uma base ortogonal de $\nu_{z} S$ na métrica $g_{\lambda}$

$$
u_{l(z)+1}^{\lambda}, \cdots, u_{m}^{\lambda}
$$

que converge suavemente para uma uma base ortogonal de $\nu_{z} S$ na métrica $g_{0}$.

\subsection{Folheações polares e isoparamétricas}

A noção de seção introduzida para ações pode ser facilmente generalizada para folheações. Nesta seção, vamos explorar uma classe particular de folheações com seção introduzida em Alexandrino [2]. Alguns exemplos e resultados são apresentados. Em particular, a estrutura local de uma dessa folheações está bem determinada; isto é uma parte do teorema de slice para essa folheações. Como será visto no teorema 3.16, as folheações polares são exemplo de folheações infinitesimalmente polares.

Considere uma folheação singular em uma variedade riemanniana. Uma seção em um ponto é uma subvariedade imersa, totalmente geodésica e completa que encontra todas as folhas de maneira ortogonal e cuja dimensão é a codimensão das folhas regulares.

Uma folheação polar é uma folheação riemanniana singular com seções, isto é, admite seção para todo ponto regular. Mais precisamente, para todo ponto regular $p$, o conjunto

$$
\Sigma_{p}:=\exp _{p}\left(\nu_{p} L_{p}\right)
$$

é uma seção.

Exemplo 2.65. Se $\mathcal{F}$ é uma folheação riemanniana $\operatorname{singular} \operatorname{com} \operatorname{codim}(\mathcal{F})=$ 1 (isto é, a codimensão das folhas regulares é 1) em uma variedade completa $M$, então $\mathcal{F}$ é polar. De fato, uma geodésica ortogonal a uma folha (e portanto a todas as outras, pois a folheação é riemanniana) é uma seção global. 


\subsection{Folheações polares e isoparamétricas}

Uma seção local de $\mathcal{F}$ é uma componente conexa na interseção de uma vizinhança tubular distinguida com uma seção de $\mathcal{F}$.

Proposição 2.66. Seja $\mathcal{F}$ uma folheação polar em uma variedade completa M. Seja $p \in M$ e $S_{p}$ slice em $p$ numa vizinhança tubular O. Então

(i) $S_{p}$ é a união das seções locais que contém $p$;

(ii) $S_{q} \subseteq S_{p}$ para $q \in S_{p}$;

(iii) $T_{q} S_{p}=T_{q} S_{q} \oplus T_{q}\left(L_{q} \cap S_{p}\right)$.

Como visto na proposição 2.54 é possível mudar a métrica da vizinhança tubular de modo que a folheação restrita a um slice permaneça riemanniana singular. A existência de seções permite esta conclusão sem mudança de métrica.

Lema 2.67. Seja $\mathcal{F}$ folheação polar em uma variedade completa $(M, g)$ e $S$ um slice. Então a folheação restrita $\mathcal{F} \cap S$ é polar com a métrica induzida.

Demonstração. A restrição é uma folheação singular. Seja $q$ um ponto em $S$ e $\gamma$ uma geodésica perpendicular à folha $L_{q}\left(\log\right.$ o perpendicular a $\left.L_{q} \cap S\right)$. Reduzindo $\gamma$ se necessário temos, pela construção do slice, que $\gamma$ está contida no slice $S_{q}$. Pela proposição 2.66, $S_{q} \subseteq S$, $\log \gamma \gamma$ está contida em $S$. Daí, como $\mathcal{F}$ é riemanniana, $\gamma$ é ortogonal a toda folha $\mathcal{F}(\log$ de $\mathcal{F} \cap S)$ que encontra. Assim $\mathcal{F} \cap S$ é riemanniana.

Finalmente, pela proposição 2.66, as seções locais para $\mathcal{F}$ que contém $q \in S$ estão em $S$. Estas seções locais são seções para $\mathcal{F} \cap S$.

Logo a folheação restrita é polar.

Folheações polares podem ser encaradas como uma generalização de folheações isoparamétricas; ver abaixo.

Exemplo 2.68. Seja $L$ uma subvariedade isoparamétrica em $M$. A partição dada por

$$
\mathcal{F}:=\left\{L_{\xi}: \xi \in \nu L \text { paralelo }\right\}
$$

é uma folheação polar.

Partições como no exemplo acima são chamadas folheações isoparamétricas.

Observação 2.69. A definição de subvariedade isoparamétrica pode ser encarada como boas condições para que, tomando as variedades paralelas como acima, se tenha uma folheação. Estas condições podem ser relaxadas; para mais detalhes, ver Toeben [39]. 


\section{Folheações infinitesimalmente polares}

Enunciamos agora um resultado de Alexandrino [3] que será útil na demonstração do teorema 3.5, além de fornecer um critério indireto para existência de seções.

Teorema 2.70. Seja $\mathcal{F}$ uma folheação riemanniana singular em uma variedade completa $M$ tal que a distribuição dos espaços normais das folhas regulares é integrável. Então $\mathcal{F}$ é uma folheação riemanniana singular com seções.

Observação 2.71. O resultado acima é uma recíproca não-trivial para um fato simples de ações polares. Seja $M_{0}$ o conjunto dos pontos regulares de uma ação. Pode-se definir uma distribuição $H$ (distribuição horizontal regular) em $M_{0}$ tal que, em cada ponto $p$,

$$
H_{p}=\nu_{p} G(p) .
$$

Se a ação for polar, temos seções $\Sigma$ em $M$ e as componentes conexas de $\Sigma \cap M_{0}$ são, por construção, subvariedades integrais para $H$. Logo $H$ é integrável.

Uma demonstração para a recíproca, na linguagem de ações, pode ser encontrada, por exemplo, em Spindola [35]. Neste sentido, temos uma forma equivalente de definir uma ação polar como uma ação isométrica tal que a distribuição dos espaços normais das órbitas principais é integrável.

Uma folheação polar é hiperpolar se as seções são flat. As subvariedades totalmente geodésicas de um espaço euclideano $\mathbb{R}^{n}$ são subespaços afins. Logo uma folheação polar em $\mathbb{R}^{n}$ é hiperpolar.

Folheações isoparamétricas podem ser definidas como a partição por préimagens de uma aplicação isoparamétrica. Para mais detalhes ver Alexandrino [2], Terng [36] ou Palais e Terng [30].

Lema 2.72. Uma folheação riemanniana singular em uma forma espacial $M(k)$ que admite uma seção é uma folheação isoparamétrica.

Observação 2.73. Se $E$ é um subespaço vetorial de $\mathbb{R}^{n}$ e $\mathcal{F}$ é uma folheação isoparamétrica de $E$. É possível induzirmos uma folheação isoparamétrica no ambiente de duas formas

(1) $\mathcal{F}^{1}$ tomando o produto das folhas de $\mathcal{F}$ com o complementar ortogonal $E^{\perp}$

(2) $\mathcal{F}^{2}$ tomando as variedades paralelas das folhas de $\mathcal{F}$.

No caso (1), as folhas da folheação original não serão folhas da folheação induzida, no entanto, toda a informação sobre a folheação induzida está 


\subsection{Reconstruindo folheações}

contida no subespaço (uma vez que o resto é trivial) e ambas têm o mesmo espaço de folhas.

No caso (2), as folhas da folheação original são folhas da folheação induzida, mas está poderá ter mais estrutura.

Exemplo 2.74. Se $\mathcal{F}$ é a folheação por círculos em $\mathbb{R}^{2} \subseteq \mathbb{R}^{3}$, seguindo a observação 2.73 , temos

(i) $\mathcal{F}^{1}$ a folheação de $\mathbb{R}^{3}$ por cilindros paralelos ao eixo $z$;

(ii) $\mathcal{F}^{2}$ a folheação de $\mathbb{R}^{3}$ por círculos centrados no eixo $z$ e paralelos ao plano $x-y$.

Lema 2.75. As construções da observação 2.73 preservam polaridade.

Demonstração. (Usamos a notação da observação 2.73.) Se $\Sigma \subseteq E$ é uma seção para $\mathcal{F}$, então $\Sigma$ é uma seção para $\mathcal{F}^{2}$ e $\Sigma \times E^{\perp}$ é uma seção para $\mathcal{F}^{1}$.

Observação 2.76. Seja $\mathcal{F}$ uma folheação isoparamétrica em $\mathbb{S}^{n-1}$. A partição de $\mathbb{R}^{n}$ por folhas homotéticas às folhas de $\mathcal{F}$ (origem é ponto singular) é uma folheação isoparamétrica de $\mathbb{R}^{n}$ que estende $\mathcal{F}$. Isto decorre da proposição 1.36, uma vez que o processo acima fornece exatamente as subvariedades paralelas das folhas.

Observação 2.77. Seja $\mathcal{F}$ uma folheação isoparamétrica em $\mathbb{R}^{n}$ tal que $x$ é uma folha. As folhas regulares estão contidas em esferas centradas em $x$. Sem perda de generalidade, pode-se assumir que $x$ é a origem. Logo $\mathcal{F}$ restrita à esfera $\mathbb{S}^{n-1}$ é isoparamétrica.

\subsection{Reconstruindo folheações}

Esta seção objetiva enunciar o teorema 2.83 - que permite reconstruir uma folheação a partir de uma folha sob determinadas hipóteses - e, como aplicação para folheações isoparamétricas, enunciar a proposição 2.85. Vamos relembrar rapidamente agumas construções em folheações; maiores detalhes podem ser obtidos, por exemplo, em Candel e Conlon [12] ou Piñeros [32].

Seja $\nabla$ uma conexão sobre uma variedade $N$ e $\gamma:[0,1] \rightarrow M$ um laço em $p=\gamma(0)=\gamma(1)$. Seja $P_{\gamma}: T_{p} N \rightarrow T_{p} N$ a isometria que leva cada $v \in T_{p} N$ em $X(1) \in T_{p} N$, onde $X$ é seu transporte paralelo ao longo de $\gamma$.

Definição 2.78. O grupo de holonomia de $N$ em $p$ é

$$
\operatorname{Hol}_{p}:=\left\{P_{\gamma}: \gamma \in \Gamma_{p}\right\}
$$




\section{Folheações infinitesimalmente polares}

onde $\Gamma_{p}$ é o conjuntos de todos os laços em $p$. Dizemos que $N$ tem holonomia trivial se o grupo de holonomia é trivial para todo ponto.

A noção de holonomia acima pode ser definida para subvariedades e diferentes conexões (por exemplo, pode-se calcular a holonomia referente à conexão normal sobre $\nu N \rightarrow N)$. É interessante obter uma noção de holonomia sobre folhas de uma folheação que forneça alguma informação sobre a geometria transversal.

Dada uma variedade $M^{n}$, uma folheação regular $\mathcal{F}$ de codimensão $p$ em $M$ é equivalente a uma distribuição integrável de $(n-p)$-planos em $M$ que dá origem a um subfibrado $E=T \mathcal{F}$ em $T M$. Existe uma sequência exata de fibrados

$$
0 \longrightarrow E \longrightarrow T M \longrightarrow Q \longrightarrow 0,
$$

onde $Q:=T M / E$. Temos $T M=E \oplus Q$ e, se $X \in \Xi(M)$ é possível escrever unicamente, seguindo esta decomposição,

$$
X=X^{e}+X^{q} .
$$

Seja $\nabla$ uma conexão afim qualquer em $Q \rightarrow M$. Para $Y \in \Gamma(Q)$ e $X \in \Xi(M)$ definimos

$$
\stackrel{\circ}{\nabla}_{X} Y:=\nabla_{X^{q}} Y+\left[X^{e}, Y\right]^{q} .
$$

Vale que $\stackrel{\nabla}{\nabla}$ é também uma conexão afim em $Q \rightarrow M$ e tal que

$$
\stackrel{\circ}{\nabla}_{X} Y=[X, Y]^{q} .
$$

Uma conexão em $Q \rightarrow M$ que verifica a equação (2.80) quando restrita a uma folha será chamada conexão de Bott. Um campo normal em uma folha regular $L$ é dito folheado se $\stackrel{\circ}{\nabla} \equiv \equiv 0$, ou seja, se é Bott-paralelo.

Observação 2.81. Se $\stackrel{\circ}{R}$ é tensor de curvatura de uma conexão de Bott $\stackrel{\circ}{\nabla}$ e $X, Y \in \Gamma(E)=\Xi(\mathcal{F})$, então $\stackrel{\circ}{R}(X, Y) \equiv 0$. Isso implica que as folhas regulares são Bott-flat.

É um fato simples de geometria riemanniana que se $R \equiv 0$, então o transporte paralelo depende apenas da classe de homotopia do caminho. Neste sentido, a holonomia mede o quanto um espaço deixa de ser flat.

Se há uma métrica $g$ em $M$ temos uma decomposição ortogonal $T M=$ $E \oplus E^{\perp}$. Podemos definir igualmente uma conexão de Bott no fibrado normal da folheação $E^{\perp} \rightarrow M$ com propriedades idênticas, em face de que a projeção $\pi: T M \rightarrow Q$ leva $E^{\perp}$ isomorficamente em $\pi\left(E^{\perp}\right)=Q$. Além disso, estes fibrados são isométricos no sentido em que se $\varphi:=\left.\pi\right|_{E^{\perp}}$, então

$$
\left.g\right|_{E^{\perp}}=\left.\varphi^{*} g\right|_{Q}
$$




\subsection{Reconstruindo folheações}

Exemplo 2.82. Seja $\mathcal{F}$ a folheação riemanniana regular dada pelas préimagens de uma submersão riemanniana $\pi: M \rightarrow B$ com $\nabla$ a conexão riemanniana de $M$. Vamos usar a notação da seção 1.5. Temos a conexão de Bott

$$
\stackrel{\circ}{\nabla}_{X} Y:=\left(\nabla_{X^{h}} Y\right)^{h}+\left[X^{v}, Y\right]^{h}
$$

e a conexão normal

$$
\nabla^{\perp}:=\left(\nabla_{X} Y\right)^{h}
$$

definidas para todo $X \in \Xi(M)$ e todo $Y$ horizontal.

Vale que $\stackrel{\circ}{\nabla}=\nabla^{\perp}$ se, e somente se, $H$ for integrável. Ou seja, pelo lema 1.30, se o tensor de O'Neill $A$ for nulo. Vale ainda que $A$ é o tensor de torção da conexão $\nabla^{\perp}$, ou seja, $\nabla^{\circ}=\nabla^{\perp}$ se, e somente se, $\nabla^{\perp}$ é simétrica.

Se $\mathcal{F}$ é uma folheação singular em $M$, dizemos que uma folha regular $L$ tem holonomia trivial se $L$ tem holonomia trivial como folha da folheação regular $\mathcal{F} \cap M_{0}$ com relação à conexão de Bott desta folheação regular.

Estamos agora em condições de enunciar o teorema. Seja $\eta_{\xi}$ a aplicação ponto-final (definição 1.19). Em Alexandrino e Toeben [6] foi demonstrado o seguinte.

Teorema 2.83. Seja $\mathcal{F}$ folheação riemanniana singular em uma variedade completa $M, L$ uma folha regular de $\mathcal{F}$ com holonomia trivial. Então

$$
\mathcal{F}=\left\{\eta_{\xi}(L): \xi \in \Xi\right\},
$$

onde $\Xi$ é o conjuntos dos campos normais folheados sobre $L$.

A demonstração deste teorema passa pelo fato das folhas regulares de folheações riemannianas singulares serem fracamente Bott-equifocais, isto é, para cada $p \in L$ folha regular, existe vizinhança $U$ de $p$ em $L$ tal que $\left.\eta_{\xi}\right|_{U}$ tem posto constante para todo $\xi$ Bott-paralelo (com a conexão de Bott no fibrado normal $E^{\perp}$ ). A partir desta propriedade (e outras considerações) se verifica um ponto central que

$$
\eta_{\xi}(U) \subseteq L_{\eta_{\xi}(p)}
$$

Esta característica das folhas regulares de folheações riemannianas singulares está relacionada a uma propriedade mais forte que constitui outra forma de se generalizar a noção de subvariedade isoparamétrica: subvariedades equifocais.

Definição 2.84. Uma subvariedade equifocal de $(M, g)$, fixada conexão $\nabla$, é uma subvariedade $L$ que tenha: fibrado normal $\nabla$-flat; aplicação ponto-final 


\section{Folheações infinitesimalmente polares}

(que está localmente definida) com posto localmente constante para vetores normais $\nabla$-paralelos; e seções, isto é, para todo $p \in L$,

$$
\Sigma_{p}=\exp _{p}\left(\nu_{p} L\right)
$$

é subvariedade de $L$ imersa, completa e totalmente geodésica.

Famílias paralelas de subvariedades equifocais (caso análogo ao exemplo 2.68) são folheações polares. Por outro lado, as folhas regulares de folheações polares são equifocais. Esta dualidade entre polaridade e equifocalidade foi explorada, por exemplo, em Alexandrino [2] e Toeben [39] e é utilizada também na demonstração dos lema 2.72, teorema 2.70, teorema 3.16 e proposição 2.66 .

O teorema 2.83 permite que possamos entender melhor folheações isoparamétricas. Em particular, uma folheação é isoparamétrica em espaços euclideanos se, e somente se, for isoparamétrica em subespaços ou esferas nos termos da proposição a seguir.

Proposição 2.85. Seja $\mathcal{F}$ uma folheação riemanniana singular em $\mathbb{R}^{n}$. Temos que $\mathcal{F}$ é isoparamétrica quando as folhas de $\mathcal{F} \cap M$ são folhas regulares de $\mathcal{F}, \mathcal{F} \cap M$ é isoparamétrica e $M$ é

(i) um subespaço vetorial; ou

(ii) uma esfera.

Demonstração. Que a folheação restrita $\mathcal{F} \cap M$ induz uma folheação isoparamétrica $\overline{\mathcal{F}}$ no ambiente segue das observações 2.76 e 2.73 (caso (2)). O fato desta folheação ser exatamente a original, isto é, $\overline{\mathcal{F}}=\mathcal{F}$, logo o resultado, segue do teorema 2.83 .

Observação 2.86. Em vista da proposição 1.39, o caso (ii) da proposição 2.85 equivale a exigir que $\mathcal{F}$ tenha folhas compactas. Por outro lado, como a folheação em questão é riemanniana, o caso $(i i)$ também equivale a dizer que o centro da esfera é uma folha. 


\section{Capítulo 3}

\section{Infinitesimalmente polar}

\subsection{Resultado principal}

Seja $\mathcal{F}$ uma folheação riemanniana singular em $M$. A restrição de $\mathcal{F}$ ao stratum regular $M_{0}$ é uma folheação riemanniana regular e os quocientes locais são variedades. Definimos $\bar{k}(z)$ como o máximo de todas as curvaturas secionais na imagem (projeção) de $z$ no quociente.

Como $M_{0}$ é subconjunto aberto e denso de $M$, todo ponto de $M$ pode ser atingido como ponto fronteiriço de $M_{0}$. Diremos que $\mathcal{F}$ satisfaz a condição de não-explosão de curvatura secional em $p \in M$ se

$$
\lim \sup _{z \rightarrow p} \bar{k}(z)<\infty, \quad z \in M_{0} .
$$

Este comportamento da curvatura no quociente está relacionado à existência de "seções infinitesimais" para $\mathcal{F}$. Este será o conteúdo do principal resultado deste texto; ver teorema 3.5.

Observação 3.2. Para ações polares ou folheações riemannianas regulares com folhas fechadas e mergulhadas, sabe-se que o quociente é um orbifold riemanniano suave, logo as curvaturas secionais são limitadas (uniformemente) em compactos.

Vamos definir mais precisamente estas seções infinitesimais.

Definição 3.3. Diremos que uma folheação riemanniana singular é infinitesimalmente polar no ponto $p$ se a folheação infinitesimal (ver seção 2.6) $\hat{\mathcal{F}}=\hat{\mathcal{F}}(p)$ for polar.

Diremos que $\mathcal{F}$ em $M$ é infitesimalmente polar se for infinitesimalmente polar em todos os pontos de $M$. Existem diversos exemplos conhecidos para esta classe de folheação. Em particular, folheações polares são infinitesimalmente polares; veremos este e outros exemplos nas seções 3.3 e 3.5. 


\section{Folheações infinitesimalmente polares}

Observação 3.4. Na notação da seção 2.6, temos

$$
\phi\left(S_{p}\right)=\phi(O) \cap E,
$$

onde $E$ é um subespaço vetorial. Este é o caso (1) da observação 2.73 e a definição 3.3 faz sentido também (vide lema 2.75) se considerada a linearização da folheação em uma vizinhança tubular (um aberto) do ponto.

A partir de agora, vamos caracterizar as folheações infinitesimalmente polares, através de condições equivalentes, como feito originalmente por Lytchak e Thorbergsson [25].

Teorema 3.5. Seja $\mathcal{F}$ uma folheação riemanniana singular em uma variedade $M$ completa. As afirmações abaixo são equivalentes:

(i) $\mathcal{F}$ satisfaz (3.1) em $p$;

(ii) $\hat{\mathcal{F}}(p)$ é polar;

(iii) $\mathcal{F}$ é localmente fechada no ponto $p$, que tem uma vizinhança $Q$ em $M$ tal que o quociente local $Q / \mathcal{F}$ é um bom orbifold riemanniano.

Em vista do teorema 3.5, passaremos a utilizar infinitesimalmente polar para toda folheação que apresentar qualquer uma das propriedades equivalentes acima.

\subsection{Demonstração}

A demonstração do teorema acima será feita por partes seguindo a estratégia abaixo.

Que $(i) \Longrightarrow($ ii $)$ será feito no teorema 3.8 através de alguns cálculos a partir de uma mudança de métrica na vizinhança tubular distinguida e resultados anteriores para folheações com seções em espaços euclideanos.

Que $(i i) \Longrightarrow($ iii $)$ será feito através de resultados conhecidos sobre o grupo de Weyl generalizado de uma seção na vizinhança tubular obtida no teorema 3.13 a partir da seção em $\hat{\mathcal{F}}$. Na observação 3.12 , clarificamos o fato de $\mathcal{F}$ ser localmente fechada (assumindo (ii)) e como o enunciado do item (iii) e a demonstração do teorema 3.13 fazem sentido. 


\subsection{Demonstração}

Que $($ iii $) \Longrightarrow($ i) é uma observação simples; ver proposição 3.15.

Lema 3.6. A condição (3.1) implica que

$$
\limsup _{z \rightarrow p} \bar{k}(z) \cdot \operatorname{dist}^{2}(z, p)=0, \quad z \in M_{0} .
$$

Teorema 3.8. Se $\mathcal{F}$ satisfaz (3.1) em p, então $\hat{\mathcal{F}}=\hat{\mathcal{F}}(p)$ é polar.

Demonstração. Considere a construção de $\left(S^{\lambda}, g_{\lambda}\right)$ da seção 2.5. Então, seguindo a observação 1.24 , temos que $h_{\lambda}$ é uma isometria de $\left(S, g_{\lambda}\right)$ para $\left(h_{\lambda}(S), \frac{1}{\lambda^{2}} g\right)$, com $h_{\lambda}(S) \subset S$. Logo, pela proposição 1.23 e equação (1.34) com $\eta=\lambda^{-1}$, temos

$$
\overline{k_{\lambda}}(z)=\overline{k^{\frac{1}{\lambda}}}\left(h_{\lambda}(z)\right)=\lambda^{2} \bar{k}\left(h_{\lambda}(z)\right) .
$$

Por outro lado, vide observação 2.64, temos que

$$
\lim _{\lambda \rightarrow 0}\left(\overline{k_{\lambda}}(z)\right)=\overline{k_{0}}(z)
$$

para curvaturas horizontais.

Veja que se $z \in S^{\lambda}$, vale que $\operatorname{dist}(z, p) \leqslant \lambda$ e $\operatorname{dist}\left(h_{\lambda}(z), p\right) \leqslant \lambda$ e, usando as equações (3.7), (3.9) e (3.10), temos que o quociente $U / \hat{\mathcal{F}}$ é flat nos pontos regulares, onde $U \subseteq \mathbb{R}^{m} \simeq T_{p} S_{p}$ é a vizinhança da origem onde está definida $\hat{\mathcal{F}}$. Além disso, sabemos que $\mathbb{R}^{m}$, logo $U$, é flat.

Logo, os dois lados da fórmula de O'Neill, ver equação (1.27), são nulos (em pontos regulares), donde a distribuição horizontal de $\hat{\mathcal{F}}$ é integrável. A existência de seção nessas condições é consequência do teorema 2.70, donde $\hat{\mathcal{F}}$ é polar (logo hiperpolar).

Observação 3.11. Como ficará claro ao final da demonstração do teorema 3.5, a equação (3.7) é também equivalente aos itens do teorema 3.5 e fornece uma caracterização técnica alternativa para polaridade infinitesimal.

Observação 3.12. O item (ii) do teorema 3.5 garante que $\hat{\mathcal{F}}$ é polar em $\mathbb{R}^{m}$ (logo hiperpolar) - de fato, $\hat{\mathcal{F}}$ polar em $\mathbb{R}^{m}$ implica que $\hat{\mathcal{F}}$ é isoparamétrica (lema 2.72). É claro que, nestas condições, $\hat{\mathcal{F}}$ é fechada (e é imediato que $\mathcal{F}$ restrita ao slice também é fechada).

Então, como discutido na seção 2.6, a folheação na vizinhança tubular é difeomorfa ao produto (em $\mathbb{R}^{n}$ ) de uma folheação fechada com uma trivial, logo é fechada. Além disso, esta parte trivial não passa ao quociente local (isto é, ao fazermos a projeção no quociente, todos os pontos na mesma folha trivial têm a mesma projeção, que depende apenas da parte isoparamétrica) de modo que basta considerar o quociente do slice.

Vale lembrar que a folheação na vizinhança tubular e a folheação linearizada, sendo difeomorfas, têm o mesmo quociente local. 


\section{Folheações infinitesimalmente polares}

Teorema 3.13. Seja $\mathcal{F}$ uma folheação riemanniana singular em $M$ completa e $p \in M$. Se $\hat{\mathcal{F}}$ é polar, então existe vizinhança $Q \subset M$ de p tal que $\mathcal{F} \cap Q$ é fechada e o quociente (local) $Q / \mathcal{F}$ é um bom orbifold riemanniano.

Demonstração. Pela observação 3.12 acima basta mostrar a conclusão do teorema para o slice $S=S_{p}$. Seja

$$
\varphi:(S, \mathcal{F}) \rightarrow(U, \hat{\mathcal{F}})
$$

a isometria que leva a folheação no slice a uma folheação polar numa vizinhança $U$ da origem em $\mathbb{R}^{m}$ (como na seção 2.6).

Como $\hat{\mathcal{F}}$ está em um espaço euclideano, temos que é hiperpolar e admite uma seção horizontal $\Sigma$ passando pela origem. Existe $\Gamma$ grupo finito de isometrias de $\Sigma$ (grupo de Weyl) que fixa a origem e tal que existe isometria

$$
\Sigma / \Gamma \rightarrow U / \hat{\mathcal{F}}
$$

Considere $N$ contido em

$$
\varphi^{-1}(\Sigma) \cap B_{\epsilon}(p) \subseteq S .
$$

Desejamos uma seção horizontal para $\mathcal{F}$, mas, em princípio, $\mathcal{F}$ pode nem admitir seções. A idéia então é definir uma nova métrica em $N$ invariante por $W=\varphi^{-1} \circ \Gamma$ de forma a tornar $N$ horizontal.

(Vamos utilizar a notação $E^{g}$ para o complemente ortogonal de um espaço vetorial $E$ em relação à métrica $g$.) Para $z \in N$, definimos

$$
\begin{aligned}
V_{z} & :=\left(T_{z} N\right)^{g_{0}} ; \\
H_{z} & :=\left(V_{z}\right)^{g} .
\end{aligned}
$$

Seja $L_{z}$ a folha de $\hat{\mathcal{F}}$ por $z$. Temos $T_{z} L_{z} \subseteq\left(T_{z} N\right)^{g_{0}}=V_{z}$, daí

$$
H_{z} \subseteq\left(T_{z} L_{z}\right)^{g},
$$

e, se $z$ é regular, vale que $H_{z}=\left(T_{z} L\right)^{g}$.

A projeção ortogonal (com respeito à métrica $g$ ) $\operatorname{pr}_{z}: T_{z} N \rightarrow H_{z}$ é um isomorfismo e definimos a métrica $\bar{g}$ por

$$
\bar{g}_{z}(\cdot, \cdot):=g_{z}\left(\operatorname{pr}_{z} \cdot \operatorname{pr}_{z} \cdot\right)
$$

em $N$.

Considere $N$ dotada da métrica $\bar{g}$. Por construção, a projeção $\pi: N \rightarrow$ $S / \mathcal{F}$ preserva distâncias na parte regular. Como $\pi$ é invariante por $W$ (uma vez que $W$ foi obtido a partir de $\Gamma$ que não altera as folhas, isto é, se $y=\omega \cdot x$ 


\subsection{Dois exemplos}

para $\omega \in W$ então $x$ e $y$ estão na mesma folha de $\mathcal{F}$ em $S$ ), os elementos de $W$ preservam comprimento de curvas na parte regular de $N$. Pela proposição 1.25 e por continuidade, os elementos de $W$ são isometrias de $N$ (com a métrica $\bar{g})$.

Então a projeção induzida

$$
N / W \rightarrow S / \mathcal{F}
$$

é um mergulho isométrico.

Observação 3.14. A distribuição $H_{z}$ da demonstrção acima é de fato suave em $z$. Considere campos linearmente independentes em $U \subseteq \mathbb{R}^{m}: v_{1}, \cdots, v_{n}$ tais que $v_{1}(z), \cdots, v_{k}(z)$ são base de $T_{z} N$. O processo (suave) de ortogonalização Gram-Schmidt fornece uma base $g_{0}$-ortogonal

$$
\begin{aligned}
& u_{1}(z)=v_{1}(z) \\
& u_{i}(z)=v_{i}(z)-\sum_{j} \frac{g_{0}\left(v_{i}, u_{j}\right)}{g_{0}\left(u_{j}, u_{j}\right)} u_{j}
\end{aligned}
$$

e, por construção, $u_{k+1}, \cdots, u_{n}$ é base $g_{0}$-ortogonal de $V_{z}$. Agora, sejam $w_{1}, \cdots, w_{n}$ a base obtida aplicando o processo Gram-Schmidt relativo à métrica $g$ à base $u_{1}, \cdots, u_{n}$, temos $w_{1}(z), \cdots, w_{k}(z)$ base de $H_{z}$.

O quociente local sendo um bom orbifold - e, portanto, quociente de uma variedade riemanniana por um grupo finito - segue que todos as curvaturas secionais em todos os pontos do quociente são limitadas. Deixamos isto registrado na próxima proposição.

Proposição 3.15. Se p tem uma vizinhança $Q$ cujo quociente $Q / \mathcal{F}$ é um bom orbifold, então $\mathcal{F}$ verifica a condição (3.1) em $p$.

\subsection{Dois exemplos}

Como mencionado antes, folheações polares são infinitesimalmente polares. Este é precisamente o enunciado do resultado abaixo; o teorema de slice para folheações riemannianas singulares com seções estabelecido em Alexandrino $[2]$.

Teorema 3.16. Seja $\mathcal{F}$ uma folheação polar em uma variedade riemanniana $M$ completa e $S_{q}$ slice em um ponto $q \in M$. Então a folheação restrita ao slice $\mathcal{F} \cap S_{q}$ é difeomorfa a uma folheação isoparamétrica em um aberto de $\mathbb{R}^{m}$, onde $m$ é a dimensão do slice $S_{q}$. 


\section{Folheações infinitesimalmente polares}

Não demonstraremos aqui este resultado; uma prova com detalhes pode ser encontrada em Piñeros [32]. A prova se utiliza de resultados anteriores para folheações riemannianas singulares integráveis, que, de fato, são polares - logo infinitesimalmente polares.

Outra classe de exemplos para folheação infinitesimalmente polar é dada pelas folheações de baixa codimensão, este é o próximo resultado.

Proposição 3.17. Seja $\mathcal{F}$ uma folheação riemanniana singular em que se tenha $\operatorname{codim}(\mathcal{F}) \leqslant 2$. Então $\mathcal{F}$ é infinitesimalmente polar.

Demonstração. O caso $\operatorname{codim}(\mathcal{F})<2$ segue do teorema 3.16 e do exemplo 2.65. Se $\operatorname{codim}(\mathcal{F})=2$, considere a folheação infinitesimal $\hat{\mathcal{F}}(p)$ em $\mathbb{R}^{n}$ para $p$ singular. Temos que a origem é uma folha e as folhas regulares estão contidas em esferas (de raio suficientemente pequeno).

Agora, se $L$ é uma folha regular, $\operatorname{codim}(L)=2 \mathrm{em} \mathbb{R}^{n}, \operatorname{logo} \operatorname{codim}(L)=1$ em uma esfera. A folheação induzida na esfera é riemanniana singular (vide lema 2.55) e tem codimensão 1, logo é polar (vide exemplo 2.65). Mas uma folheação é polar em $\mathbb{R}^{n}$ se, e somente se, é polar na esfera, vide proposição 2.85, donde concluímos que $\mathcal{F}$ é infinitesimalmente polar.

\subsection{Completude variacional}

Nesta seção, vamos explorar alguns resultados sobre folheações sem pontos horizontalmente conjugados e ações variacionalmente completas (definição 2.11) que são, vide proposição 3.18, essencialmente o mesmo assunto. $\mathrm{Na}$ seção 3.5, utilizaremos os conceitos aqui desenvolvidos no intuito de mostrar que ações variacionalmente completas dão origem a folheações infinitesimalmente polares.

Seja $M$ uma variedade riemanniana e $\mathcal{F}$ uma folheação riemanniana singular em $M$. Dizemos que $\mathcal{F}$ não tem pontos horizontalmente conjugados quando para toda folha $L$ e toda geodésica $\gamma$ que encontra $L$ perpendicularmente, todo campo $L$-Jacobi (vide seção 1.3) ao longo de $\gamma$ que é tangente a uma outra folha de $\mathcal{F}$ (diferente de $L$ ) é tangente a todas as folhas que $\gamma$ encontra.

Proposição 3.18. Seja $G$ um grupo de Lie agindo em uma variedade riemanniana completa $M$ por isometrias e seja $\mathcal{F}$ a folheação riemanniana singular em $M$ induzida pela ação de $G$. Então a ação de $G$ é variacionalmente completa se, e somente se, $\mathcal{F}$ não tem pontos horizontalmente conjugados.

Demonstração. Seja $L$ uma folha de $\mathcal{F}$, ou seja, uma órbita de $G$. Seja $\gamma$ uma geodésica de $M$ que encontra $L$ perpendicularmente e $J$ um campo $L$-Jacobi sobre $\gamma$ que é tangente a uma folha diferente de $L$. 


\subsection{Completude variacional}

Se a ação é variacionalmente completa, então

$$
J=\left.X^{\xi}\right|_{\gamma}
$$

donde $J$ é tangente a todas as órbitas que $\gamma$ encontra, logo vale que $\mathcal{F}$ não tem pontos horizontalmente conjugados.

Se $\mathcal{F}$ não tem pontos horizontalmente conjugados, então $J$ é tangente a todas as órbitas. Seja $t_{0}$ tal que $\gamma\left(t_{0}\right)$ não é ponto focal de $L$ e seja $X$ restrição a $\gamma$ de um campo de Killing que verifica $X\left(t_{0}\right)=J\left(t_{0}\right)$ (é sempre possível obter $X$, vide observação 3.19 abaixo). Agora temos, pelo lema 1.15, que $X$ é campo de Jacobi. Mais precisamente, $X$ é $L$-Jacobi (ver observação 3.20) e $\log o Y:=X-J$ também é $L$-Jacobi. Mas $Y\left(t_{0}\right)=0$ em um ponto que não é $L$-focal, portanto, $Y \equiv 0$ em $\gamma$.

Logo $J$ é restrição de um campo de Killing induzido pela ação e temos que esta é variacionalmente completa.

Observação 3.19. Temos que $J\left(t_{0}\right)$ é um vetor tangente em $\gamma\left(t_{0}\right)$. A folheação $\mathcal{F}$ é induzida pela ação isométrica de $G$. Pela transitividade de $\Xi(\mathcal{F})$, temos que existe campo $X^{\xi}$, para algum $\xi \in \mathfrak{g}$, como na equação (2.4) que coincide com $J\left(t_{0}\right)$ em $\gamma\left(t_{0}\right)$. Mas, como a ação é isométrica, o fluxo de $X^{\xi}$ é um isometria, logo este é um campo de Killing.

Observação 3.20. Usando o lema 1.15 e a observação 3.19 é claro que a variação associada ao campo $X=X^{\xi}$ é

$$
\gamma_{s}(t)=\mu(\exp s \xi, \gamma(t))
$$

onde $\mu: G \times M \rightarrow M$ é a ação isométrica de $G$ em $M$.

Considere $h:=\exp s \xi$ e $\mu^{h}:=\mu(h, \cdot)$ que é uma isometria e $\gamma_{s}(0)=$ $\mu^{h}(\gamma(0))$. Agora, para todo $s, d \mu^{h}$ leva $\gamma^{\prime}(0)$ em $\gamma_{s}^{\prime}(0)$.

Se $d \mu^{h}$ leva $T_{\gamma(0)} L$ em $T_{\gamma_{s}(0)} L$, sabendo que $\gamma$ é ortogonal à folha $L$, temos que todas as curvas da variação são ortogonais à $L$ e temos o desejado, isto é, $X$ é $L$-Jacobi. Como $d \mu^{h}$ é isomorfismo, basta mostrar que $d \mu^{h}\left(T_{\gamma(0)} L\right) \subseteq$ $T_{\gamma_{s}(0)} L$ para concluir a igualdade.

De fato, um vetor em $T_{\gamma(0)} L$ tem a forma $\left.\frac{d}{d \epsilon} \mu(\exp \epsilon \eta, \gamma(0))\right|_{\epsilon=0}$ para algum $\eta \in \mathfrak{g}$. Aplicando $d \mu^{h}$ temos

$$
\begin{aligned}
\left.\frac{d}{d \epsilon} \mu^{h}(\mu(\exp \epsilon \eta, \gamma(0)))\right|_{\epsilon=0} & =\left.\frac{d}{d \epsilon} \mu(h \cdot \exp \epsilon \eta, \gamma(0))\right|_{\epsilon=0} \\
& =\left.\frac{d}{d \epsilon} \mu\left(h \cdot \exp \epsilon \eta \cdot h^{-1}, \mu(h, \gamma(0))\right)\right|_{\epsilon=0} \\
& =\left.\frac{d}{d \epsilon} \mu\left(\exp (\epsilon A d(h) \eta), \gamma_{s}(0)\right)\right|_{\epsilon=0} .
\end{aligned}
$$




\section{Folheações infinitesimalmente polares}

(Para uma justificativa da última passagem, ver, por exemplo, a seção 1.3 de Alexandrino e Bettiol [5].) Mas $A d(h) \eta \in \mathfrak{g}$, donde

$$
d \mu^{h}\left(T_{\gamma(0)} L\right) \subseteq T_{\gamma_{s}(0)} L,
$$

e isto conclui a observação.

O conceito de completude variacional foi introduzido em Bott [9] como uma classe de ações isométricas "suficientemente ricas" ([9], p. 260) para que o núcleo focal de $L$ (campos $L$-Jacobi não-nulos que se anulam em um ponto focal) fosse formado inteiramente por campos que fossem estendíveis a campos globais induzidos pela ação. Naquela ocasião, o autor desejava aplicar desigualdades de Morse e era relevante saber a dimensão do núcleo focal. Neste sentido uma ação é variacionalmente completa quando "produz campos de Jacobi suficientes, ao longo de geodésicas, para determinar a multiplicidade dos pontos focais das órbitas" (Conlon [17], p. 149).

A riqueza das ações variacionalmente completas se traduz também em um quociente bom. Na introdução de Bott e Samelson [10], os autores afirmam pensar completude variacional "intuitivamente" ([10], p. 965) como a ausência de pontos conjugados no quociente da ação. Uma forma interessante de pensar isso é entender que a condição sem pontos horizontalmente conjugados é uma condição transversal, isto é, pode ser formulada em termos da estrutura do quociente. Ausência de pontos horizontalmente conjugados se traduz na não-existência de pontos conjugados no quociente. Vamos ilustrar essa idéia no exemplo simples a seguir.

Exemplo 3.21. Seja $\pi: M \rightarrow B$ uma submersão riemanniana e $\mathcal{F}$ a folheação regular definida pelas fibras $\pi^{-1}(b)$ para todo $b \in B$. Suponha que $\mathcal{F}$ é sem pontos horizontalmente conjugados.

Agora seja $J$ campo de Jacobi em $\alpha$ em $B$ associado a uma variação $\alpha_{s}(t):[0,1] \times[0,1] \rightarrow B$ e tal que $J(\alpha(0))=0=J(\alpha(1))$. Podemos levantar as geodésicas (vide lema 1.26) obtendo uma variação $\tilde{\alpha}_{s}(t):[0,1] \times[0,1] \rightarrow M$ com campo de Jacobi associado $\tilde{J}$. Por construção, $\tilde{J}$ é vertical em $\tilde{\alpha}(0)$ e $\tilde{\alpha}(1)$. Mas, pela hipótese de $\mathcal{F}$ ser sem pontos horizontalmente conjugados, isso implica que $\tilde{J}$ é vertical em todo ponto de $\tilde{\alpha}$. Logo $J \equiv 0$.

Concluímos que $B$ não tem pontos conjugados.

Observação 3.22. Pelo raciocínio do exemplo 3.21 acima, concluímos também a recíproca: se $B$ não tem pontos conjugados, então $\mathcal{F}$ é sem pontos horizontalmente conjugados.

Vamos agora enunciar dois resultados que podem ser encontrados em Lytchak e Thorbergsson [24]. Naquela ocasião, os autores estudaram ações 


\subsection{Completude variacional}

variacionalmente completas em espaços de curvatura não-negativa. Este é o caso do espaço euclideano e, como já foi visto, folheações riemannianas singulares em variedades admitem uma linearização da folheação restrita ao slice, contexto onde estes resultados podem ser usados.

Vale notar que resultados na mesma direção que estes foram obtidos anteriormente em Gorodski e Thorbergsson [21] para espaços simétricos; ver teorema 2.14 .

Proposição 3.23. Seja $M$ uma variedade riemanniana completa e $\mathcal{F}$ uma folheação riemanniana singular em $M$ que admite seções flat. Então $\mathcal{F}$ não tem pontos horizontalmente conjugados.

O resultado acima é uma generalização da proposição 2.12 - originalmente obtida em Conlon [16] - à luz da proposição 3.18.

Teorema 3.24. Seja $M$ uma variedade riemanniana completa e $\mathcal{F}$ uma folheação riemanniana singular em $M$ sem pontos horizontalmente conjugados. Então, se $M$ tem curvatura secional não-negativa, $\mathcal{F}$ é hiperpolar.

Vamos apresentar um roteiro informal das idéias da demonstração do teorema 3.24 seguindo Lytchak e Thorbergsson [24].

Seja $\gamma: \mathbb{R} \rightarrow M$ uma geodésica horizontal completa tal que $\gamma(0)$ é regular. Considere $\tilde{\mathcal{E}}$ o conjunto dos campos $L_{\gamma(c)}$-Jacobi sobre $\gamma$ para algum $c \in \mathbb{R}$. E seja $\mathcal{E} \subset \tilde{\mathcal{E}}$ o conjunto dos campos tais que

$$
J(t) \in T_{\gamma(t)} L_{\gamma(t)}
$$

para todo $t \in \mathbb{R}$. Vale que, para todo $t \in \mathbb{R}$,

$$
\tilde{V}_{t}:=T_{\gamma(t)} L_{\gamma(t)}=\{J(t): J \in \mathcal{E}\} .
$$

Se $\gamma(t)$ é regular, então $\tilde{V}_{t}$ e seu complemento ortogonal $\tilde{H}_{t}$ definem espaços verticais e horizontais análogos aos de uma submersão riemanniana (vide seção 1.5), donde a equação (1.29) faz sentido e podemos considerar o tensor $A_{\gamma^{\prime}(t)}$ nos pontos regulares.

A estratégia é definir um tensor horizontal sobre toda a geodésica que estenda $A$ aos pontos singulares (que são isolados em $\gamma$ ) e portanto obter uma medida da integrabilidade da distribuição horizontal (vide lema 1.30). Este dado, em vista do teorema 2.70, permite concluir a existência de seções.

Para definir um tensor horizontal em toda a geodésica os autores seguem técnicas apresentadas em Wilking [42]. Define-se

$$
V^{\prime}{ }_{t}:=\left\{D_{t} J(t): J \in \mathcal{E}, J(t)=0\right\}
$$




\section{Folheações infinitesimalmente polares}

e verifica-se que este espaço é trivial nos pontos regulares para o caso em questão. Mais precisamente, vale que

$$
V_{t}:=\tilde{V}_{t}+V^{\prime}{ }_{t}
$$

é uma distribuição suave sobre $\gamma$, de dimensão constante e que coincide com o espaço tangente das órbitas $T_{\gamma(t)} L_{\gamma(t)}$ nos pontos regulares.

Seja $H_{t}$ o complemento ortogonal de $V_{t}$ e considere $X=X^{v}+X^{h}$ a decomposição única de um campo ao longo de $\gamma$ em relação à decomposição ortogonal $T_{\gamma(t)} M=V_{t} \oplus H_{t}$.

Define-se um novo tensor horizontal

$$
A_{t}(X(t)):=\left(D_{t}\left(X^{h}\right)(t)\right)^{v}+\left(D_{t}\left(X^{v}\right)(t)\right)^{h},
$$

que coincide com $A_{\gamma^{\prime}(t)}$ onde este está definido, ou seja, nos pontos regulares.

Observe que os $V_{t}$ e $H_{t}$ sao fibras, respectivamente, de dois fibrados (sobre $\mathbb{R}) V_{\gamma}$ e $H_{\gamma}$. A conexão $\nabla$ em $(M, g)$ induz conexões $D_{t}^{v}$ e $D_{t}^{h}$, respectivamente, sobre estes fibrados. Em Wilking [42], mostra-se que um vetor $Y$ é da forma $Y=J^{h}$, onde $J$ é um campo de Jacobi sobre $\gamma$ se

$$
D_{t}^{h} D_{t}^{h} Y+\left(R\left(Y, \gamma^{\prime}\right) \gamma^{\prime}\right)^{h}-3 A_{t} A_{t} Y=0
$$

Esta equação é melhor interpretada à luz do tensor de O'Neill. Considere ação de $G$ em $M$ e $B:=M / G$ o quociente. Sejam $\pi: M \rightarrow B$ a projeção usual, $p \in M$ um ponto regular e $\{X, Y\}$ base ortonormal de um 2-plano $\sigma_{p}$ em $H_{p}$. Então, pela equação (1.31),

$$
\begin{aligned}
k_{B}\left(d \pi_{p}\left(\sigma_{p}\right)\right) & =k_{M}\left(\sigma_{p}\right)+3\left\|A_{X} Y\right\|^{2} \\
& =R(X, Y, X, Y)+3\left\|A_{X} Y\right\|^{2} \\
& =g\left((R(Y, X) X)^{h}-3 A_{X} A_{X} Y, Y\right) .
\end{aligned}
$$

Lembramos que $l:=\operatorname{dim}\left(H_{t}\right)-1$ não depende de $t$. Considere $X_{1}, \cdots, X_{l}$ campos horizontais e $D_{t}^{h}$-paralelos sobre $\gamma$ tais que $\left\{\gamma^{\prime}, X_{1}, \cdots, X_{l}\right\}$ seja ortonormal em todo ponto (logo base de $H_{t}$ ). Se $\Phi: \mathbb{R}^{l} \rightarrow H_{\gamma}$ leva

$$
\left(v_{1}, \cdots, v_{l}\right) \mapsto \sum v_{i} X_{i}
$$

então $\Phi$ é uma bijeção entre curvas em $\mathbb{R}^{l}$ e vetores horizontais perpendiculares ao longo de $\gamma$. Para uma curva $\alpha: \mathbb{R} \rightarrow \mathbb{R}^{l}$ definimos, a partir da equação (3.29), um operador auto-adjunto em $\mathbb{R}^{l}$

$$
\left.\mathcal{R}(t) \alpha(t):=\Phi^{-1}\left(R\left(Y(t), \gamma^{\prime}(t)\right) \gamma^{\prime}(t)\right)^{h}-3 A_{t} A_{t} Y(t)\right),
$$

onde $Y:=\Phi \circ \alpha$.

Observe que o lado direto da equação (3.27) é sempre positivo se duas condições são verificadas: 


\subsection{Completude variacional}

(i) $M$ tem curvatura não-negativa;

(ii) a distribuição $H$ é não-integrável.

Como visto na seção 1.5, a integrabilidade da distribuição horizontal é medida pelo tensor $A$, neste caso, $H$ é integrável na parte regular se, e somente se, $A_{t} \equiv 0$. Nestas condições, da equação (3.29), segue que

$$
(R(Y, X) X)^{h}-3 A_{X} A_{X} Y>0
$$

e $\log O$

$$
\langle\mathcal{R}(t) \alpha(t), \alpha(t)\rangle>0
$$

para toda curva $\alpha$ e todo real $t$.

Define-se uma forma índice

$$
I_{a}^{b}(\alpha, \beta):=\int_{a}^{b}\left\langle\alpha^{\prime}(t), \beta^{\prime}(t)\right\rangle-\langle\mathcal{R}(t) \alpha(t), \beta(t)\rangle d t
$$

onde $\alpha$ e $\beta$ são curvas contínuas que se anulam nos extremos e tais que suas derivadas são quadrado-integráveis.

Como visto, as hipóteses do teorema 3.24 levam à equação (3.31), que implica que o índice de $I_{a}^{b}$ é positivo em um grande intervalo $[a, b] \subset \mathbb{R}$ (ver observação 3.32 abaixo).

Observação 3.32. Se $A_{t_{0}} \neq 0$, então existe $x \in \mathbb{R}^{l}$ tal que

$$
\int_{t_{0}-1}^{t_{0}+1}\langle\mathcal{R}(t) x, x\rangle d t=C_{0}>0 .
$$

Agora, para $N$ suficientemente grande, é possível encontrar uma função real suave $\phi: \mathbb{R} \rightarrow \mathbb{R}$ tal que

(i) $\phi(t)=0$ se $t \notin[a, b]$;

(ii) $\phi(t)=1$ se $t \in\left[t_{0}-1, t_{0}+1\right]$; e

(iii) $\int_{a}^{b}\left(\phi^{\prime}(t)\right)^{2} d t<C_{0}$,

onde $a:=t_{0}-N$ e $b:=t_{0}+N$. Tomando $\alpha(t):=\phi(t) x$, temos

$$
I_{a}^{b}(\alpha, \alpha)<0
$$




\section{Folheações infinitesimalmente polares}

Existem resultados análogos à teoria clássica de Morse (teorema 1.18) que garantem, nestas condições, solução não-trivial de

$$
\alpha^{\prime \prime}+\mathcal{R} \alpha=0
$$

com

$$
\alpha(a)=0=\alpha(c)
$$

para um $c \in(a, b)$.

O campo horizontal $Y:=\Phi \circ \alpha$ para $\alpha$ solução obtida acima verifica a equação (3.26) e se anula em $\gamma(a)$ e $\gamma(c)$, que podemos assumir pontos regulares. Se $J$ é o campo de Jacobi em $\gamma$ definido pelas condições iniciais $J(a)=0$ e $\left(D_{t} J\right)(a)=D_{t}^{h} Y(a)$ então $J^{h}$ e $Y$ são soluções da mesma equação diferencial com mesmas condições iniciais, donde são iguais. Como $J$ verifica as condições da proposição 1.21, temos que é $L_{\gamma(a)^{-}}$-Jacobi. Ou seja, existe $J \in \tilde{\mathcal{E}}$ tal que $J^{h}=Y=\Phi \circ \alpha$.

Estamos em condições de enunciar o seguinte.

Lema 3.33. Seja $\mathcal{F}$ uma folheação não-polar em uma variedade $M$ com curvatura não-negativa. Então existe ao menos uma geodésica horizontal com pontos horizontalmente conjugados.

Seguindo o discutido acima, resumido no lema 3.33, em vista do teorema 2.70 e da hipótese de ausência de pontos horizontalmente conjugados em $\mathcal{F}$, chegamos na integrabilidade da distribuição normal da folheação - o roteiro acima implica que, para todo $X$ horizontal $\left.A_{X}\right|_{M_{0}} \equiv 0$ - e logo na polaridade de $\mathcal{F}$. As técnicas acima também garantem que a seção é flat.

\subsection{Caso variacionalmente completo}

Nesta seção, vamos exibir outro exemplo de polaridade infinitesimal. São folheações sem pontos horizontalmente conjugados, um classe que, quando provém de ações isométricas, é equivalente ao conceito de completude variacional (proposição 3.18).

Teorema 3.34. Folheações sem pontos horizontalmente conjugados são infinitesimalmente polares.

O teorema 3.34 pode ser visto como uma generalização do teorema 3.24, que, com hipóteses adicionais, garante que a folheação é polar (logo infinitesimalmente polar). 


\subsection{Caso variacionalmente completo}

Primeiramente vamos passar aos lemas abaixo, onde utilizamos a notação introduzida na seção 2.5. A idéia por trás dos próximos lemas é que a mudança de métrica

$$
g \longrightarrow \tilde{g} \longrightarrow \frac{1}{\lambda^{2}} \tilde{g} \longrightarrow g_{\lambda} \longrightarrow g_{0}
$$

não altera a propriedade sem pontos horizontalmente conjugados.

A primeira mudança, vide proposição 2.54 , não provoca alterações no quociente - logo não poderia "criar pontos conjugados na base" o que, pela discussão antes do exemplo 3.21 deveria acontecer para que $\mathcal{F}$ deixasse de ser sem pontos horizontalmente conjugados.

A segunda mudança, vide equação (1.32), não altera a conexão de Riemann. Além disso, por serem métricas homotéticas, é claro que a noção de ortogonalidade não se altera. Na terceira passagem, vide observação $1.24 \mathrm{e}$ proposição 2.47, como já ressaltado na demonstração do teorema 3.8, temos uma isometria

$$
\left(S, g_{\lambda}\right) \rightarrow\left(S^{\lambda}, \frac{1}{\lambda^{2}} \tilde{g}\right)
$$

que leva folhas em folhas. Logo "variação por geodésicas ortogonais" em $\left(S, g_{\lambda}\right)$ é sempre a imagem isométrica de uma "variação por geodésicas ortogonais" em $\left(S^{\lambda}, \frac{1}{\lambda^{2}} \tilde{g}\right)$, que, por sua vez, é exatamente o mesmo que "variação por geodésicas ortogonais" em $\left(S^{\lambda}, \tilde{g}\right)$.

Finalmente, a idéia da última passagem é que cada geodésica na métrica $g_{0}$ pode ser encarada como limite de uma sequência de geodésicas nas métricas $g_{\lambda}$ quando $\lambda \rightarrow 0$ e que tal convergência não altera a ausência de pontos conjugados.

Diremos que uma geodésica é regular se todos os seus pontos forem regulares. Entender o caso para estas geodésicas será suficiente para demonstrar o teorema 3.34 (ver lema 3.40). Neste caso podemos nos restringir a vizinhanças onde a folheação é localmente regular logo localmente dada por submersão e podemos tornar as idéias acima de forma mais rigorosa seguindo o exemplo 3.21 .

Seja $p \in M$ singular, $O$ sua vizinhança tubular de Molino, $S$ slice que contém $p$ e $\tilde{\mathcal{F}}:=\mathcal{F} \cap S$ a folheação restrita a $S$.

Lema 3.35. Se $\mathcal{F}$ é uma folheação riemanniana singular em $(M, g)$ sem pontos horizontalmente conjugados. Então, geodésicas horizontais regulares de $\tilde{\mathcal{F}}$ em $(S, \tilde{g})$ não têm pontos horizontalmente conjugados.

Demonstração. Seja $\tilde{\gamma}$ segmento de geodésica horizontal a $\tilde{\mathcal{F}}$ que contém apenas pontos regulares. Pela proposição 2.54, para $q \in S$ com a métrica $\tilde{g}$, o espaço normal da folha $\tilde{L}_{q}:=L_{q} \cap S$ é tangente a $S$. Assim, uma geodésica que encontra $\tilde{L}_{q}$ perpendicularmente permanece em $S$, bem como variações 


\section{Folheações infinitesimalmente polares}

por geodésicas ortogonais estão em $S$, logo um campo $\tilde{L}_{q^{-}}$Jacobi em $O$ é um campo $\tilde{L}_{q^{-}}$Jacobi em $S$.

Agora, pela proposição 2.54 e observação 3.4 , temos

$$
(S, \tilde{g}) / \tilde{\mathcal{F}} \simeq(O, \tilde{g}) / \mathcal{F} \simeq(O, g) / \mathcal{F} .
$$

Como $\tilde{\gamma}$ é regular, considerando uma vizinhança regular da folheação, o lema segue pela discussão antes do exemplo 3.21, da hipótese de $\mathcal{F}$ ser sem pontos conjugados e da equação (3.36).

Lema 3.37. Se $\mathcal{F}$ é uma folheação riemanniana singular em $(M, g)$ sem pontos horizontalmente conjugados. Então geodésicas horizontais regulares de $\tilde{\mathcal{F}}$ em $\left(S, g_{\lambda}\right)$ não têm pontos horizontalmente conjugados.

Demonstração. Este lema segue diretamente do lema 3.35 em vista de "variação por geodésicas ortogonais" ser o mesmo para $\tilde{g}$ e $g_{\lambda}$ numa vizinhança regular.

Agora, vamos enunciar, sem demonstração, o seguinte resultado técnico ([25], lema 5.5).

Lema 3.38. Seja $\mathcal{F}$ uma folheação riemanniana singular em $(M, g)$. Seja $\left(g_{n}\right)$ sequência de métricas em $M$ que converge suavemente

$$
g_{n} \rightarrow g
$$

e tais que $\mathcal{F}$ permanece riemanniana em $\left(M, g_{n}\right)$. Seja $\left(\gamma_{n}\right)$ sequência de curvas tais que $\gamma_{n}:[a, b] \rightarrow M$ é geodésica horizontal para métrica $g_{n} e$

$$
\gamma_{n} \rightarrow \gamma
$$

onde $\gamma:[a, b] \rightarrow M$ é geodésica horizontal regular em $(M, g)$ tal que $\gamma(b)$ não é ponto focal de $L_{\gamma(a)}$.

Então, se cada $\gamma_{n}$ não tem pontos horizontais conjugados, $\gamma$ também não tem pontos horizontais conjugados.

Lema 3.39. Se $\mathcal{F}$ é uma folheação riemanniana singular em $(M, g)$ sem pontos horizontalmente conjugados. Então, geodésicas horizontais regulares de $\tilde{\mathcal{F}}$ em $\left(S, g_{0}\right)$ não têm pontos horizontalmente conjugados.

Demonstração. Seja $\gamma_{0}:[a, b] \rightarrow M$ geodésica regular em $\left(S, g_{0}\right)$ de forma que $\gamma(b)$ não seja ponto focal de $L_{\gamma(a)}$ (pode ser feito escolhendo intervalo $[a, b]$ conveniente). 


\subsection{Caso variacionalmente completo}

Considere $\gamma_{\lambda}:[a, b] \rightarrow M$ geodésica em $S$, em relação à métrica $g_{\lambda}$, definida unicamente por $\gamma_{\lambda}(0)=p$ e $\gamma_{\lambda}^{\prime}(0)=\gamma_{0}^{\prime}(0)$. Temos, por construção, que

$$
\gamma_{\lambda} \rightarrow \gamma_{0}
$$

Pelo lema 3.37, $\gamma_{\lambda}$ não tem pontos horizontalmente conjugados (para métrica $g_{\lambda}$ ) e logo, pelo lema $3.38, \gamma_{0}$ não tem pontos horizontais conjugados (para métrica $g_{0}$ ). Lembre que, pelo lema $2.60, g_{\lambda}$ converge suavemente para $g_{0}$.

Como a escolha de $\gamma_{0}$ foi arbitrária, concluímos o desejado.

Sabemos que $\tilde{\mathcal{F}}$ em $\left(S, g_{0}\right)$ é isométrica a $\hat{\mathcal{F}}$ em $(U$, can), onde $U$ é uma vizinhança da origem de um espaço euclideano $\mathbb{R}^{m}$ e can é a métrica euclideana canônica; como visto na seção 2.6.

Lema 3.40. Se $\hat{\mathcal{F}}$ não é polar, então existe pelo menos uma geodésica horizontal regular em $U$ com pontos horizontalmente conjugados.

O lema acima segue de raciocínio similar ao roteiro do teorema 3.24. Neste caso, porém, em vista do teorema 3.5, sabemos que a curvatura do quociente explode para certos caminhos. A idéia é a seguinte. Para alguma geodésica horizontal regular $\gamma$ em $U$ (que é uma vizinhança convexa da origem) definida em $[\bar{a}, \bar{b}]$, com tensor $A_{t}$ definido sobre $\gamma$ e $t_{0} \in[\bar{a}, \bar{b}]$ tal que $A_{t_{0}} \neq 0$. Procedendo em analogia à observação 3.32 , tem-se, neste caso, $\int_{t_{0}-\epsilon}^{t_{0}+\epsilon}\langle\mathcal{R}(t) x, x\rangle d t$ suficientemente grande para obter um intevalo pequeno $[a, b] \subset[\bar{a}, \bar{b}]$ onde a forma índice é negativa. Seguindo o roteiro do teorema 3.24 chega-se a conclusão mais forte, neste caso, que existe geodésica horizontal regular com pontos horizontalmente conjugados.

Demonstração do teorema 3.34. Se $\hat{\mathcal{F}}$ não é polar, pelo lema 3.40, há uma geodésica horizontal regular em $U$ com pontos horizontalmente conjugados. Segue que há geodésica horizontal regular em $\left(S, g_{0}\right)$ com pontos horizontalmente conjugados para $\tilde{\mathcal{F}}$. Mas o lema 3.39 garante que tal não acontece, donde $\hat{\mathcal{F}}$ é polar e $\mathcal{F}$ é infinitesimalmente polar.

Terminamos esta seção enunciando, sem demonstração, uma descrição geométrica global de folheações sem pontos horizontalmente conjugados obtida em Nowak [28].

Teorema 3.41. Uma folheação riemanniana singular é sem pontos horizontalmente conjugados se, e somente se, os pontos singulares são exatamente os pontos focais das folhas. 


\section{Folheações infinitesimalmente polares}

Em uma folheação riemanniana qualquer, pontos singulares são sempre pontos focais de uma folha. Mas, em geral, pontos focais de folhas regulares podem estar em folhas regulares, como no exemplo trivial abaixo.

Exemplo 3.42. Na esfera, pontos antipodais são conjugados na métrica usual. Seja $\mathcal{F}$ a folheação (regular) na esfera em que as folhas são pontos. Qualquer geodésica na esfera é uma geodésica horizontal para $\mathcal{F}$. Logo os pontos conjugados são horizontalmente conjugados. Em particular, um ponto (folha regular) é focal em relação a outro (folha regular).

\subsection{A estrutura de $M / \mathcal{F}$}

Nesta seção, desenvolveremos mais resultados para entender melhor a estrutura global do quociente $M / \mathcal{F}$. Apresentaremos também uma versão do teorema 3.5 para ações: o teorema 3.45. Por fim, na proposição 3.50 ilustramos o caso em que o quociente não possui estrutura de orbifold com uma classe de exemplos que surge naturalmente.

Primeiramente, seguindo Alexandrino [4], observamos que a discussão feita na seção 2.4 para entender o quociente de uma folheação regular se estende para o caso de folheações infinitesimalmente polares com pequenas modificações.

Seja $\mathcal{F}$ uma folheação infinitesimalmente polar em $M$. Todo ponto de $M$ tem uma vizinhança onde $\mathcal{F}$ é difeomorfa ao produto de uma folheação isoparamétrica com uma folheação trivial (este produto é polar). Seja $\left(U_{\alpha}\right)$ cobertura de $M$ por vizinhanças desta forma. Como visto na demonstração do teorema 3.5 (ver demonstração do teorema 3.13) é possível encontrar, em $U_{\alpha}$, uma seção local $\sigma_{\alpha}$ com uma métrica tal que a distância entre dois pontos em $\sigma_{\alpha}$ seja igual a distância entre as folhas que os contém na métrica de $M$.

A interseção das folhas singulares com uma seção local é formada por um número finito de hiperfícies totalmente geodésicas (difeomorfas a subvariedades focais da folheação linearizada) chamadas paredes.

Uma câmara de Weyl $C_{\alpha}$ em $\sigma_{\alpha}$ é a união de uma componente conexa $C$ de $M_{0} \cap \sigma_{\alpha}$ com as paredes contidas em $\partial C$. Uma câmara de Weyl é sempre um conjunto convexo.

Se $U_{\alpha} \cap U_{\beta} \neq 0$, é possível encontrar abertos $V_{\alpha}^{\alpha} \subseteq \sigma_{\alpha}, V_{\beta}^{\alpha} \subseteq \sigma_{\beta}$ e uma isometria

$$
\varphi_{\beta \alpha}: V_{\alpha}^{\alpha} \cap C_{\alpha} \rightarrow V_{\beta}^{\alpha} \cap C_{\beta},
$$

onde $C_{\alpha}$ e $C_{\beta}$ são câmaras de Weyl em $\sigma_{\alpha}$ e $\sigma_{\beta}$. Através de reflexões nas paredes, é possível obter uma isometria

$$
\varphi_{\beta \alpha}: V_{\alpha}^{\alpha} \rightarrow V_{\beta}^{\alpha}
$$


chamada holonomia (singular).

Assim como na seção 2.4 , os $\varphi_{\beta \alpha}$ agindo em

$$
\sigma:=\bigcup \sigma_{\alpha}
$$

formam um pseudogrupo $\Phi$. A noção de equivalencia da definição 2.39 se aplica também neste caso.

Concluímos que o quociente global de uma folheação infinitesimalmente polar é um orbifold riemanniano (não necessariamente bom).

Lema 3.43. Seja $\mathcal{F}$ uma folheação riemanniana singular fechada em $M$ completa. Uma folha $L_{x}$ é um ponto orbifold no quociente global $M / \mathcal{F}$ se, e somente se, $\mathcal{F}$ é infinitesimalmente polar em $x$.

Demonstração. Sabemos do teorema 3.5 que se $\mathcal{F}$ é infinitesimalmente polar em $x$, então $O / \mathcal{F}$ é um bom orbifold riemanniano para uma vizinhança tubular $O$ de $x$. Por outro lado, como as folhas de $\mathcal{F}$ não se acumulam, a imagem (projeção) de $O$ no quociente global $M / \mathcal{F}$ é o quociente de $O / \mathcal{F}$ por no máximo um grupo finito - logo é também um bom orbifold riemanniano.

Agora se $L_{x} \in M / \mathcal{F}$ é um ponto orbifold, então é claro que $\bar{k}(x)<\infty$ e, pelo teorema 3.5 temos que é $\mathcal{F}$ é infinitesimalmente polar em $x$.

O resultado abaixo é decorrência direta da definição de orbifold, da discussão no início desta seção e da última frase na demonstração do lema 3.43.

Proposição 3.44. Seja $\mathcal{F}$ folheação riemanniana singular fechada em $M$ completa. O quociente global $M / \mathcal{F}$ é um orbifold riemanniano se, e somente se, $\mathcal{F}$ é infinitesimalmente polar.

O lema 3.43 nos coloca em condições de enunciar uma versão do teorema 3.5 para ações. Seja $K$ um subgrupo fechado do grupo de isometrias de uma variedade riemanniana $M$. Considere $x \in M$ e o grupo de isotropia $K_{x}$ agindo no espaço normal $H_{x}$ da órbita $K(x)$. O conjunto de pontos com órbita principal $M_{0}$ é aberto e denso em $M$. Considere

$$
B:=M / K, \quad B_{0}:=M_{0} / K
$$

e $y:=K(x) \in B$ a imagem de $x$ no espaço de órbitas.

Teorema 3.45. As afirmações abaixo são equivalentes:

(i) $\lim \sup _{z \rightarrow y} \bar{k}(z)<\infty, \quad z \in B_{0}$;

(ii) $\limsup \sup _{z \rightarrow y} \bar{k}(z) \cdot \operatorname{dist}^{2}(y, z)=0, \quad z \in B_{0}$; 


\section{Folheações infinitesimalmente polares}

(iii) a ação de $K_{x}$ em $H_{x}$ é polar;

(iv) uma vizinhança de $y \in B$ é um bom orbifold riemanniano.

Comparando o item (iii) do teorema acima com o item (ii) do teorema 3.5, podemos entender de forma mais precisa em que sentido a folheação linearizada em um ponto (vide seção 2.6) é análoga, no cenário de folheações, à representação isotrópica (vide seção 2.2), no cenário de ações.

Abaixo, temos alguns dos exemplos mais simples do quociente global de folheações por folhas paralelas a uma superfície em $\mathbb{R}^{3}$.

Exemplo 3.46. Se $\mathcal{F}$ é a partição de $\mathbb{R}^{3}$ pelos planos afins paralelos a determinado plano $\pi$, então $\mathcal{F}$ é uma folheação regular e $M / \mathcal{F}$ é $\mathbb{R}$.

Exemplo 3.47. Seja $\mathcal{F}$ a partição de $\mathbb{R}^{3}$ por esferas centradas na origem (isto é, partição pelas subvariedades paralelas a uma esfera qualquer centrada na origem). Então $\mathcal{F}$ é isoparamétrica e a única singularidade (variedade focal) é a origem. O quociente pode ser visto como uma reta qualquer que passa pela origem com pontos simétricos identificados.

Logo $M / \mathcal{F}$ é um bom orbifold (também uma variedade-com-bordo, ver exemplo 2.34).

Exemplo 3.48. Se $\mathcal{F}$ é a partição de $\mathbb{R}^{3}$ por cilindros paralelos de mesmo eixo. É fácil ver que $\mathcal{F}$ é o produto de uma folheação por esferas (círculos) em $\mathbb{R}^{2}$ por uma folheação trivial em $\mathbb{R}$. Logo o quociente é o mesmo do exemplo anterior: uma semi-reta.

Observação 3.49. Considere a folheação riemanniana singular em $\mathbb{R}^{n+1}$ dada por $n$-esferas,

$$
L_{p}=\left\{x \in \mathbb{R}^{n+1}:|x|=|p|\right\},
$$

com a origem como única singularidade. E considere $\mathbb{R}^{n+m}=\mathbb{R}^{n+1} \times \mathbb{R}^{m-1}$ com folheação $\bar{L}$ dada pelo produto da folheação por esferas em com uma parte trivial, isto é, para $(p, q) \in \mathbb{R}^{n+1} \times \mathbb{R}^{m-1}$,

$$
\bar{L}_{(p, q)}=\left\{L_{p} \times \mathbb{R}^{m-1}\right\} .
$$

Temos que $\bar{L}$ é uma folheação riemanniana singular e quociente global é o mesmo do exemplo 3.48 .

Podemos concluir que, dados $n, m>0$ quaisquer, existe uma folheação riemanniana singular $\mathcal{F}$ em uma variedade completa $M=\mathbb{R}^{n+m}$ com

$$
\operatorname{dim}(\mathcal{F})=n \text { e } \operatorname{codim}(\mathcal{F})=m
$$

tal que o quociente global $M / \mathcal{F}$ é um bom orbifold. 
Concluímos esta dissertação com uma proposição que não está em Lytchak e Thorbergsson [25]. Este resultado ilustra o caso em que o teorema 3.5 permite concluir que o quociente em questão não é um orbifold.

Proposição 3.50. Seja $\mu: \mathbb{S}^{1} \times M \rightarrow M$ uma ação isométrica e efetiva. Então o quociente $M / \mathbb{S}^{1}$ é um orbifold se, e somente se, o stratum singular $\Sigma^{0}$ verifica $\operatorname{codim}\left(\Sigma^{0}\right) \leqslant 2$.

Antes da demonstração da proposição, vamos dar dois exemplos simples.

Exemplo 3.51. O espaço de órbitas não é um orbifold para

$$
\begin{aligned}
\mu: \mathbb{S}^{1} \times \mathbb{C}^{2} & \rightarrow \mathbb{C}^{2} \\
\left(\zeta,\left(z_{1}, z_{2}\right)\right) & \mapsto\left(\zeta z_{1}, \zeta z_{2}\right) .
\end{aligned}
$$

Basta observar que, neste caso, o stratum singular é igual à folha singular 0 , que tem codimensão $4 \mathrm{em} \mathbb{C}^{2}$.

Observe que esta ação é isométrica, porém, não é polar. Considere $p=$ $(1,0)$ que é regular, temos que $\nu_{p}\left(\mathbb{S}^{1}(p)\right)$ é o 3 -plano que passa e por $p$ e cujo vetor normal é $(i, 0)$. Agora o ponto $q=(1,1)$ pertence a este plano, mas $T_{q}\left(\mathbb{S}^{1}(q)\right)$ é a reta paralela ao vetor $(i, i)$ que passa por $q$. Logo a interseção do candidato (único) a seção com as órbitas não é sempre ortogonal (pois $(i, 0)$ e $(i, i)$ não são paralelos), ou seja, a ação não é polar.

Exemplo 3.52. O exemplo acima pode ser generalizado trivialmente para

$$
\begin{aligned}
\mu: \mathbb{S}^{1} \times\left(\mathbb{C}^{2} \times \mathbb{R}^{n}\right) & \rightarrow\left(\mathbb{C}^{2} \times \mathbb{R}^{n}\right) \\
\left(\zeta,\left(z_{1}, z_{2}\right), p\right) & \mapsto\left(\zeta z_{1}, \zeta z_{2}, p\right) .
\end{aligned}
$$

Demonstração da proposição 3.50. No caso em que $\Sigma^{0} \neq 0$ e $\operatorname{codim}\left(\Sigma^{0}\right)=0$, temos $M=\Sigma^{0} \simeq M / \mathbb{S}^{1}$, logo vale (trivialmente) a proposição.

Tome $x_{0} \in \Sigma^{0}$ e considere sua vizinhança tubular com a métrica dada na proposição 2.54. Considere a folheação riemanniana singular $\tilde{\mathcal{F}}:=\mathcal{F} \cap S_{x_{0}}$ e sua linearização $\hat{\mathcal{F}}=A^{-1} \exp ^{-1}(\tilde{\mathcal{F}})$ em $U \subseteq \mathbb{R}^{m}$.

Observe que $0=A^{-1} \exp ^{-1}\left(x_{0}\right)$ é folha de $\hat{\mathcal{F}}$ e as folhas regulares estão localmente a uma distância constante de 0 , isto é, se $L$ é uma folha regular de $\hat{\mathcal{F}}$ então

$$
B_{\epsilon}(0) \cap L \neq 0 \Longrightarrow L \subseteq B_{\epsilon}(0)
$$

para $\epsilon$ pequeno.

Por outro lado a folha 0 está em $\hat{\Sigma}^{0}:=A^{-1} \exp ^{-1}\left(\Sigma^{0}\right)$ que é o stratum minimal de $\hat{\mathcal{F}}$ e logo as folhas regulares estão contidas em tubos centrados em $\hat{\Sigma}^{0}$ (vide lema 2.51). 


\section{Folheações infinitesimalmente polares}

Então temos que, se $L$ é uma folha regular,

$$
L \subseteq B_{\epsilon}(0) \cap \operatorname{Tub}_{\epsilon}\left(\hat{\Sigma}^{0}\right) \subseteq E^{\perp},
$$

onde $E^{\perp}$ é o subespaço vetorial dado por $\left(T_{0} \hat{\Sigma}^{0}\right)^{\perp}$. Em particular, $\operatorname{dim} L=1$.

Afirmação 1: $\hat{\mathcal{F}}$ é isoparamétrica em $\mathbb{R}^{n}$ se, e somente se, for isoparamétrica em $E^{\perp}$. Este fato segue da proposição 2.85 .

A proposição 3.44 diz que o quociente será orbifold se, e somente se, $\hat{\mathcal{F}}$ for isoparamétrica. Lembramos que 0 é o único ponto singular de $\hat{\mathcal{F}}$ em $E^{\perp}$.

Afirmação 2: $\left.\hat{\mathcal{F}}\right|_{E^{\perp}}$ é isoparamétrica se, e somente se, $\operatorname{codim}(L) \leqslant$ 1 para folhas regulares. Se $\operatorname{codim}(L)=0$, é trivial. $\operatorname{Se} \operatorname{codim}(L)=1$, temos retas como seções, segue que $\hat{\mathcal{F}}$ é isoparamétrica. Por outro lado, se $\operatorname{codim}(L)>1$ e $\hat{\mathcal{F}}$ é isoparamétrica, temos uma seção $\Sigma$ com $\operatorname{dim} \Sigma>1$ e a interseção dos pontos singulares de $\hat{\mathcal{F}}$ com $\Sigma$ tem de ser uma hiperfície em $\Sigma$ - contrariando que 0 é o único ponto singular.

Como $\operatorname{dim} L=1$, as afirmações 1 e 2 nos dão que $M / \mathbb{S}^{1}$ é um orbifold se, e somente se, $\hat{\mathcal{F}}$ é isoparamétrica se, e somente se,

$$
\operatorname{dim} E^{\perp}=\operatorname{codim}\left(\hat{\Sigma}^{0}\right) \leqslant 2
$$

se, e somente se,

$$
\operatorname{codim}\left(\hat{\Sigma^{0}}\right)=\operatorname{codim}\left(\Sigma^{0}\right) \leqslant 2
$$




\section{Referências}

[1] J.F. Adams: Lectures on Lie groups, W.A. Benjamin, 1969.

[2] M.M. Alexandrino: Singular Riemannian foliations with sections, Illinois Journal of Mathematics 48, 2004.

[3] M.M. Alexandrino: Proofs of conjectures about singular Riemannian foliations, Geometria Dedicata 119, 2006.

[4] M.M. Alexandrino: On polar foliations and fundamental group, preprint, 2010. Disponível em http://arxiv.org/abs/1012.0926.

[5] M.M. Alexandrino, R.G. Bettiol: Introduction to Lie groups, adjoint action and some generalizations, preprint, 2010. Disponível em http://arxiv.org/abs/0901.2374.

[6] M.M. Alexandrino, D. Toeben: Equifocality of singular Riemannian foliations, Proceedings of the American Mathematical Society 136, 2008.

[7] M. Berger: Encounter with a geometer, part II, Notices of the American Mathematical Society 47, 2000.

[8] J.E. Borzellino, V. Brunsden: Determination of the topological structure of an orbifold by its group of orbifold diffeomorphisms, Journal of Lie Theory 13, 2003.

[9] R. Bott: An application of the Morse theory to the topology of Liegroups, Bulletin de la Société Mathématique de France 84, 1956.

[10] R. Bott, H. Samelson: Applications of the theory of Morse to symmetric spaces, American Journal of Mathematics 80, 1958. Correction to..., American Journal of Mathematics 83, 1961.

[11] M. Bridson, A. Haefliger: Metric spaces of non-positive curvature, Grundlehren der mathematischen Wissenschaften 319, Springer-Verlag, 1999. 


\section{Folheações infinitesimalmente polares}

[12] A. Candel, L. Conlon: Foliations II, Graduate studies in mathematics 60, American Mathematical Society, 2003.

[13] M.P. do Carmo: Geometria riemanniana, Projeto Euclides, IMPA, 1988.

[14] J.L.O. Chamorro: Subvariedades isoparamétricas do espaço euclidiano, dissertação de mestrado sob orientação de C. Gorodski no IME da USP, 2008.

[15] I. Chavel: Riemannian geometry: a modern introduction, Cambridge studies in advanced mathematics 98, Cambridge University Press, 2006.

[16] L. Conlon: Variational completeness and K-transversal domains, Journal of Differential Geometry 5, 1971.

[17] L. Conlon: A class of variationally complete representations, Journal of Differential Geometry 7, 1972.

[18] R. Correa da Silva: Folheações riemannianas singulares, tese de doutorado sob orientação de Francisco Rui de Almeida no IME da USP, 1993.

[19] J.J. Duistermaat, J.A.C. Kolk: Lie groups, Universitext, SpringerVerlag, 2000.

[20] C. Gorodski: A survey on the geometry of isometric actions, Matemática Contemporânea 28, 2005.

[21] C. Gorodski, G. Thorbergsson: Variationally complete actions on compact symmetric spaces, Journal of Differential Geometry 62, 2002.

[22] D. Joyce: On manifolds with corners, preprint, 2009. Disponível em http://arxiv.org/abs/0910.3518.

[23] I. Kolar, J. Slovak, P. Michor: Natural operations in differential geometry, corrected electronic version, 1999. Disponível em http://www . emis.de/monographs/KSM/.

[24] A. Lytchak, G. Thorbergsson: Variationally complete actions on nonnegatively curved manifolds, Illinois Journal of Mathematics 51, 2007.

[25] A. Lytchak, G. Thorbergsson: Curvature explosion in quotients and applications, Journal of Differential Geometry 85, 2010. 


\section{Referências}

[26] I. Moerdijk, J. Mrcun: Introduction to foliations and Lie groupoids, Cambridge studies in advanced mathematics 91, Cambridge University Press, 1999.

[27] P. Molino: Riemannian foliations, Progress in mathematics 73, Birkhauser, 1988. Com apêndices por G. Cairns; Y. Carriere; E. Ghys; E. Salem; e V. Sergiescu.

[28] E. Nowak: Singular riemannian foliations: exceptional leaves, tautness, preprint, 2008.

[29] B. O'Neill: The fundamental equations of a submersion, Michigan Mathematical Journal 13, 1966.

[30] R.S. Palais, C.-L. Terng: Critical point theory and submanifold geometry, Lectures notes in mathematics 1353, Springer-Verlag, 1988.

[31] P. Petersen: Riemannian geometry, Graduate texts in mathematics 171, Springer-Verlag.

[32] D.A.C. Piñeros: Sobre as folheações e o teorema de slice para folheações riemannianas singulares com seções, dissertação de mestrado sob orientação de M.M. Alexandrino no IME da USP, 2008.

[33] I. Satake: On a generalization of the notion of manifold, Proceedings of the National Academy of Sciences 42, 1956.

[34] J.-P. Serre: Tores maximaux des groupes de Lie compacts, Séminaire Sophus Lie 1, 1955.

[35] F.L. Spindola: Grupos de Lie, ações próprias e a conjectura de PalaisTerng, dissertação de mestrado sob orientação de M.M. Alexandrino no IME da USP, 2008.

[36] C.-L. Terng: Isoparametric submanifolds and their Coxeter groups, Journal of Differential Geometry 21, 1985.

[37] G. Thorbergsson: Singular Riemannian foliations and isoparametric submanifolds, Milan Journal of Mathematics 78, 2010.

[38] W.P. Thurston: The geometry and topology of threemanifolds, electronic version, 2002. Disponível em http://library.msri.org/books/gt3m. 


\section{Folheações infinitesimalmente polares}

[39] D. Toeben: Parallel focal structure and singular Riemannian foliations, Transactions of the American Mathematical Society 358, 2006.

[40] Q.-M. Wang: Isoparametric functions on Riemannian manifolds, Mathematische Annalen 277, 1987.

[41] M. Weilandt: Isospectral metrics on weighted projective spaces, tese de doutorado sob orientação de Dorothee Schüth na Universidade Humboldt de Berlim, 2010.

[42] B. Wilking: A duality theorem for Riemannian foliations in nonnegative sectional curvature, Geometric and Functional Analysis 17, 2007. 


\section{Índice remissivo}

ação, 20

ação hiperpolar, 22

ação isométrica, 22

ação polar, 22

ação própria, 21

ação variacionalmente completa, 23

bom orbifold, 29

Bott

conexão de, 44

curvatura secional, 4

espaço simétrico, 23

explosão de curvatura, 47

folheação, 26

folheação hiperpolar, 42

folheação infinitesimalmente polar, 47

folheação polar, 40

folheação regular, 31

folheação riemanniana, 32

folheação riemanniana singular, 28

folheação sem pontos horizontalmente conjugados, 52

folheação singular, 26

folheação transnormal, 28

folheações isoparamétricas, 41

holonomia regular, 32

holonomia singular, 63

holonomia trivial, 43

isoparamétrica

família, 17 folheação, 41

função, 16

hiperfície, 17

subvariedade, 16

Jacobi

campo $L-, 9$

campo de, 8

Killing

campo de, 8

métrica $\bar{g}, 50$

métrica $\hat{g}, 36$

métrica $\tilde{g}, 36$

métrica $g^{\eta}, 14$

métrica $g_{\lambda}, 37$

mergulho isométrico, 11

Molino

conjectura de, 29

lema de, 35

vizinhança de, 34

O'Neill

fórmula de, 14

tensor de, 14

orbifold, 33

placa, 34

ponto conjugado, 8

ponto focal, 9

pseudogrupo, 32

quociente (de uma ação), 29

quociente (de uma folheação), 34 


\section{Folheações infinitesimalmente polares}

representação isotrópica, 23

seção (de uma ação), 22

seção (de uma folheação), 40

seção local, 41

slice, 35

submersão riemanniana, 13

subvariedade equifocal, 45

subvariedade isoparamétrica, 16

teorema de slice para folheações polares, 51

teorema do toro maximal, 24

teorema principal, 48

teorema principal para ações, 64

variedade focal, 16

variedade paralela, 16

vizinhança tubular, 34

Weyl

câmara de, 25, 62

grupo de, 25 